\title{
Atomic-Scale Investigation of the Structural and Electronic Properties of Cobalt-Iron Bimetallic Fischer-Tropsch Catalysts
}

Ahmed S. M. Ismail, ${ }^{\dagger}{ }^{\oplus}$ Marianna Casavola, ${ }^{\dagger}$ B⿺ Boyang Liu, ${ }^{\#}$ Alexandre Gloter, ${ }^{\S}$ Tom W. van Deelen, ${ }^{\dagger}$ Marjan Versluijs, ${ }^{\dagger}$ Johannes D. Meeldijk, ${ }^{\dagger}$ Odile Stéphan, ${ }^{\S}$ Krijn P. de Jong, and Frank M. F. de Groot* ${ }^{*}+$ (1)

${ }^{\dagger}$ Inorganic Chemistry and Catalysis, Debye Institute for Nanomaterials Science, Utrecht University, 3584 CG Utrecht, The Netherlands

${ }^{\ddagger}$ Experimental Biophysics and Nanotechnology group, Physics Department, King’s College London, WC2R 2LS London, United Kingdom

\#Department of Physics, Stockholm University, SE-106 91 Stockholm, Sweden

${ }^{\S}$ Laboratoire de Physique des Solides, Universitè Paris-Sud, F-91405 Orsay, France

Supporting Information

ABSTRACT: Supported cobalt and iron catalysts are important for many catalytic processes. It is believed that combining both elements in a bimetallic catalyst would result in a synergetic catalytic activity during Fischer-Tropsch Synthesis. However, the nature of the interaction between the two elements and its effect on their catalytic properties are not well understood. In the present work, we report the synthesis of carbon-nanotube-supported $\mathrm{Co}-\mathrm{Fe}$ alloy catalysts using colloidal chemistry methods. The ratios of $\mathrm{Co}-\mathrm{Fe}$ and their homogeneous distribution in the supported nanoparticles were confirmed using local and bulk characterization techniques. We observed the formation of $\mathrm{Co}-\mathrm{Fe}$ Janus-like alloy structure and a change of their elemental distribution at

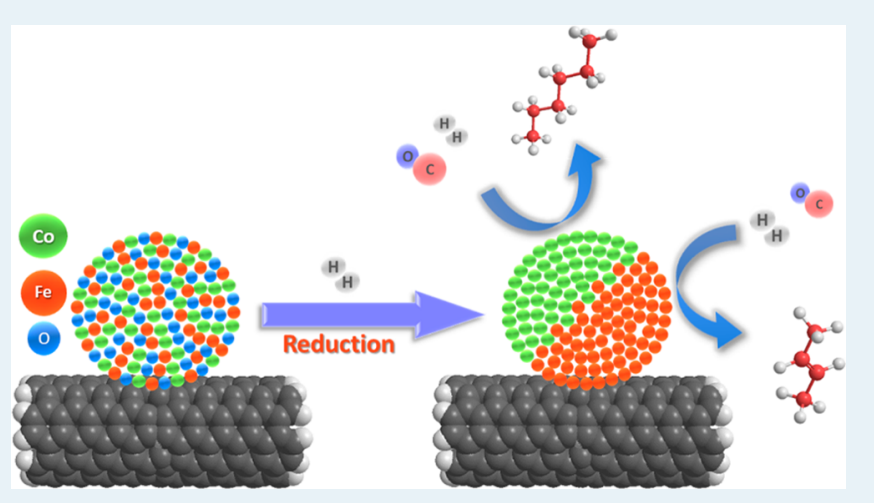
the nanoparticle scale upon reduction. The bimetallic catalyst showed reasonable Fischer-Tropsch catalytic activity and selectivity to $\mathrm{C}_{5+}$ hydrocarbons when compared with monometallic iron and cobalt catalysts. Finally, we found that the active phase of the $\mathrm{Co}-\mathrm{Fe}$ bimetallic species during catalysis depends on the catalytic conditions.

KEYWORDS: Fischer-Tropsch, cobalt, iron, bimetallic catalyst, colloidal synthesis, alloys

\section{INTRODUCTION}

Cobalt and iron have been extensively studied for over a century because of their catalytic properties for a number of important reactions, such as Fischer-Tropsch synthesis, ${ }^{1}$ ammonia synthesis, ${ }^{2}$ the water-gas shift reaction, ${ }^{3}$ carbon nanotube (CNT) growth, ${ }^{4}$ and many other processes. ${ }^{5}$ In addition to their catalytic behavior, the ferromagnetic properties of cobalt ferrite $\left(\mathrm{CoFe}_{2} \mathrm{O}_{4}\right)$ nanoparticles have made them an important candidate for magnetic device applications, including loudspeakers, electric motors, and microwave components. $^{6}$

Fischer-Tropsch (FT) synthesis is a good example where catalysts of cobalt and iron have been successful. The process is used to obtain synthetic fuel from a mixture of $\mathrm{CO}$ and $\mathrm{H}_{2}$, socalled syngas, originating mainly from alternative feedstocks such as coal, biomass, or natural gas. ${ }^{7}$ Cobalt and iron are used differently under the syngas stream at elevated temperatures. Supported cobalt catalysts are utilized mainly during the lowtemperature FT synthesis, where the reaction temperature ranges from 220 to $260{ }^{\circ} \mathrm{C}$ and the products tend to be higher chain $\left(\mathrm{C}_{5_{+}}\right)$hydrocarbons. Cobalt is considered the best catalyst for the gas to liquid process (GTL) because of its high activity, stability, and selectivity to paraffins at high $\mathrm{H}_{2} / \mathrm{CO}$ ratios. It is widely believed that $\mathrm{FT}$ proceeds over cobalt catalysts in their metallic states because traces of metallic cobalt have been detected during and after the FT process. ${ }^{8}$ In addition, it was reported that the catalytic performance of cobalt catalysts in FT was independent of the particle size for catalysts with particle sizes above $6 \mathrm{~nm}$. ${ }^{9}$ This study also suggested that cobalt particles with sizes smaller than $6 \mathrm{~nm}$ exhibit low activity and higher methane selectivity. On the other hand, supported iron catalysts can be employed under different $\mathrm{CO}: \mathrm{H}_{2}$ ratios and higher-temperature FT conditions $\left(320-360{ }^{\circ} \mathrm{C}\right)$ where they give higher selectivity to shortcarbon-chain olefins $\left(\mathrm{C}_{2}-\mathrm{C}_{4}\right)$, which can later be polymerized into higher-chain hydrocarbons. Another direction is the

Received: October 27, 2018

Revised: April 19, 2019

Published: June 7, 2019 
utilization of iron in lower-temperature FT where it gives high catalytic activity and has higher selectivity to paraffin wax that can be hydrocracked into diesel fuel or refined wax products. ${ }^{7}$

In addition to monometallic cobalt and iron catalysts, bimetallic catalysts of both elements have also been a topic of research and discussion for a long time. This interest stems from the prediction that a bimetallic cobalt-iron catalyst will give synergetic catalytic properties. In addition, it is believed that the presence of iron will reduce the catalyst costs and the presence of a low percentage of cobalt can enhance the iron reducibility and activity at lower temperatures. ${ }^{10}$ Therefore, reaching optimal $\mathrm{Fe}-\mathrm{Co}$ compositions can lead to a costeffective and active FT catalyst for a selective reaction at lower temperature. However, the research conducted on CoFe bimetallic catalysts so far led to contradictory results that made it difficult to make a final and clear conclusion on the structural composition and the catalytic behavior of $\mathrm{Co}-\mathrm{Fe}$ bimetallic catalysts. $^{11}$

Some recent studies conducted on $\mathrm{CoFe} / \mathrm{CNT}$ suggested that by increasing the percentage of $\mathrm{Fe}$ in the bimetallic catalyst up to $0.5 \%$ by weight, higher $\mathrm{CO}$ conversion could be reached without affecting the selectivity of cobalt to $\mathrm{C}_{5+}$ hydrocarbons. ${ }^{12}$ However, adding higher percentages of iron to the bimetallic catalyst will result in a gradual decrease in the catalyst activity and higher selectivity to methane and less $\mathrm{C}_{5+}$ hydrocarbons. It was also noted that alcohols yield increased by addition of iron. Another study claimed that cobalt and iron supported on carbon nanofibers gave synergetic effects and that $\left(\mathrm{Co}_{75} \mathrm{Fe}_{25} / \mathrm{CNF}\right)$ had the highest production of $\mathrm{C}_{5+}$ hydrocarbons and a lower selectivity to methane and carbon dioxide. ${ }^{13}$ Both studies agreed that addition of iron to cobalt enhances the dispersion of cobalt on the support and that the presence of cobalt facilitates the reduction of iron to its metallic state. With no clear results and agreement on the catalyst behavior during FT, and without revealing the cobalt and iron chemical nature and specific roles in the process, it is difficult to assess the potential use of this bimetallic catalytic system for FT.

The majority of the previously reported work on supported metal oxide catalysts have employed the incipient wetness impregnation method for the preparation of catalysts. ${ }^{14}$ This method involves the introduction of the active metal as a precursor to the support followed by consecutive drying steps to get rid of any remaining liquid solvent followed by a calcination step, where undesirable salts such as nitrates are decomposed and removed as nitrogen oxides under the influence of high temperature and carrier gas. Although this method is relatively easy and suitable for industrial applications, controlling the particle size and morphology of the supported metal nanoparticles is cumbersome. It is believed that the interaction between metal precursor and the hydroxide groups on the support is strong and may result in the formation of regions of high concentration of metal nanoparticles and other regions with low concentrations of metal nanoparticles. ${ }^{15}$

One possible novel approach to control the chemical and physical properties of supported metal catalysts is by using colloidal synthesis routes. Colloidal chemistry has been used extensively for decades to prepare metal oxide nanocrystals with almost monodisperse particle size and a high homogeneous morphological structure. ${ }^{16}$ Such approach for nanocrystals synthesis has gained a lot of interest in many research fields such as for gas sensing, ${ }^{17}$ semiconductors, ${ }^{18,19}$ and recently catalysis. ${ }^{20}$ For heterogeneous catalysis, this synthesis approach promises great advantages in control of particle size, geometry, and chemistry of the synthesized catalysts over the conventional incipient wetness impregnation method. ${ }^{21}$

In this work, we tried to further understand the chemical and structural nature of the cobalt-iron bimetallic catalysts and to establish a link between the catalyst properties and its FischerTropsch catalytic activity. First, we explored the synthesis of CNT-supported cobalt-iron bimetallic catalysts using nonhydrolytic colloidal synthesis routes. Nonfunctionalized carbon nanotubes have been chosen as a support in this work on the model bimetallic catalysts as they have a weak metal-support interaction in comparison with metal oxide supports. This weak interaction will improve the distribution of the active metal nanoparticles over the support and increase their surface area, which in turn has been found to enhance the active metal reducibility. $^{22,24}$ These supports are also known to be thermally and chemically stable. We then investigated the valence states and structural evolution of bimetallic catalysts of different compositions as well as their FT catalytic performance at different temperatures and $\mathrm{CO}: \mathrm{H}_{2}$ ratio.

\section{EXPERIMENTAL METHODS}

Catalyst Preparation. The cobalt ferrite $\left(\mathrm{CoFe}_{2} \mathrm{O}_{4}\right)$ nanocrystals were synthesized using the nonhydrolytic colloidal process. ${ }^{25}$ In this process, coordination compounds of cobalt(II) and iron(III) acetylacetonate were used as precursors to synthesize $\mathrm{CoFe}_{2} \mathrm{O}_{4}$ spherical nanocrystals (Figure S1).

To make $\mathrm{Co}_{30} \mathrm{Fe}_{70}$ nanocrystals (reproduced from ref 26), 2 $\mathrm{mmol}$ of $\mathrm{Co}(\mathrm{acac})_{2}$ (Sigma-Aldrich) was added to $40 \mathrm{~mL}$ of diphenyl ether, $20 \mathrm{mmol}$ of 1,2-hexadecanediol (SigmaAldrich), $10 \mathrm{~mL}$ of oleic acid, and $10 \mathrm{~mL}$ of oleylamine (Sigma-Aldrich). The mixture was degassed for $30 \mathrm{~min}$ and then heated to $140{ }^{\circ} \mathrm{C}$ under inert atmosphere for another 30 $\mathrm{min}$. Then, $4 \mathrm{mmol}$ of $\mathrm{Fe}(\mathrm{acac})_{3}$ (Sigma-Aldrich) dissolved in $20 \mathrm{~mL}$ of diphenyl ether (Alfa Aesar) was added dropwise to the mixture containing the cobalt precursor. The mixture temperature was then raised rapidly $(\sim 10 \mathrm{~min})$ to $260{ }^{\circ} \mathrm{C}$ and kept at reflux for $30 \mathrm{~min}$ before being cooled to room temperature. Afterward, the mixture was washed with ethanol, centrifuged at $400 \mathrm{rpm}$ for $5 \mathrm{~min}$, and redispersed in toluene. The process was repeated multiple times to remove any remaining solvents and unreacted materials after the reaction. For the synthesis of $\mathrm{Co}_{50} \mathrm{Fe}_{50}$ nanocrystals, the concentration of $\mathrm{Co}(\mathrm{acac})_{2}$ was increased to $4 \mathrm{mmol}$ while the concentration of $\mathrm{Fe}(\mathrm{acac})_{3}$ was kept constant. For $\mathrm{Co}_{70} \mathrm{Fe}_{30}$ nanocrystals, the concentration for $\mathrm{Co}(\mathrm{acac})_{2}$ was $8 \mathrm{mmol}$ at constant $\mathrm{Fe}(\mathrm{acac})_{3}$ concentration. Finally, to make $\mathrm{Co}_{10} \mathrm{Fe}_{90}$ nanocrystals, we used $\mathrm{Co}(\mathrm{acac})_{2}$ and $\mathrm{Fe}(\mathrm{acac})_{3}$ with a concentration ratio of $1: 4$ mmol, respectively.

Monometallic iron and cobalt nanocrystals were also fabricated as references for the comparison of the catalytic performance. To fabricate $5 \mathrm{~nm}$ iron oxide nanocrystals, a degassed mixture of $3 \mathrm{mmol}$ of $\mathrm{Fe}(\mathrm{CO})_{5}$ and $2 \mathrm{~mL}$ of octadecene was rapidly injected to another mixture containing $0.67 \mathrm{mmol}$ of oleyamine and $10 \mathrm{~mL}$ of octadecene at $220^{\circ} \mathrm{C}$ under vacuum. The mixture was then kept in reflux for $15 \mathrm{~min}$. Afterward, the suspension was cooled, and toluene was added to extract the nanocrystals, which were then purified by repeated precipitation in isopropanol, centrifugation, and redispersion in toluene. Finally, the nanoparticles were dispersed in $15 \mathrm{~mL}$ of octadecene. The monometallic cobalt 
nanocrystals were designed according to the procedure reported by van Deelen et al., ${ }^{23}$ where $7.5 \mathrm{~mL}$ of 1,2 dichlorobenzene (Sigma-Aldrich, 99\%, anhydrous) was added to $65 \mathrm{mg}$ of oleic acid in a three-necked flask under nitrogen atmosphere and degassed at $100{ }^{\circ} \mathrm{C}$. The mixture was then heated to $174{ }^{\circ} \mathrm{C}$ while it was magnetically stirred. Then the cobalt precursor containing $270 \mathrm{mg}$ of dicobalt octacarbonyl (Acros Organics, 95\%, stabilized) and $1.5 \mathrm{~mL}$ of $1,2-$ dichlorobenze was rapidly injected to the flask, and the mixture was kept in reflux for $20 \mathrm{~min}$. Afterward, the suspension was cooled, and nanocrystals were then purified by repeated precipitation in 2-propanol, centrifugation, and redispersion in $n$-hexane. Finally, the nanoparticles were dispersed in $3 \mathrm{~mL}$ of toluene.

To make a catalyst with $10 \%$ by weight metal loading, $8 \mathrm{~mL}$ of the freshly prepared cobalt ferrite nanocrystals dispersed in toluene was then added to $15 \mathrm{~mL}$ of 1 -octadocene (SigmaAldrich). The mixture was then briefly sonicated for a couple of minutes to improve the dispersion of nanocrystals and then added to $250 \mathrm{mg}$ of carbon nanotubes (CNT) (Baytubes C $150 \mathrm{HP}$ from Bayer Material Science), which were presieved to 200-400 $\mu \mathrm{m}$. The mixture was then degassed for $15 \mathrm{~min}$ at $120{ }^{\circ} \mathrm{C}$ and heated for $30 \mathrm{~min}$ at $200{ }^{\circ} \mathrm{C}$ under inert atmosphere and stirring. After the supernatant was cooled, it was safely disposed, and the mixture was washed with acetone and hexane (3:1) multiple times to remove any remaining ligands. The catalysts were then dried in a static air oven at 120 ${ }^{\circ} \mathrm{C}$ for $3 \mathrm{~h}$ followed by another $3 \mathrm{~h}$ drying process under vacuum at $120{ }^{\circ} \mathrm{C}$.

For catalytic activity comparison, another set of bimetallic cobalt-iron catalysts were prepared via the incipient wetness coimpregnation method, where carbon nanotubes (CNT, Baytubes C $150 \mathrm{HP}$ from Bayer Material Science) were first presieved to $200-400 \mu \mathrm{m}$ and functionalized using a liquidphase oxidation procedure. Typically, the CNT were dried for $2 \mathrm{~h}$ at $120{ }^{\circ} \mathrm{C}$, then added to a round flask containing $96 \%$ $\mathrm{HNO}_{3}$ (Sigma-Aldrich) and the mixture was kept in reflux for $3 \mathrm{~h}$ at $150{ }^{\circ} \mathrm{C}$. Afterward, the suspension was cooled, and the $\mathrm{CNT}$ were filtered off and washed with water until the $\mathrm{pH}$ was close to neutral. The materials were then dried in a static air oven at $120{ }^{\circ} \mathrm{C}$ for $12 \mathrm{~h}$.

The cobalt nitrate hexahydrate $\left(\mathrm{Co}\left(\mathrm{NO}_{3}\right)_{2} \cdot 6 \mathrm{H}_{2} \mathrm{O}\right)$ (SigmaAldrich) and iron nitrate nonahydrate $\left(\mathrm{Fe}\left(\mathrm{NO}_{3}\right)_{3} \cdot 9 \mathrm{H}_{2} \mathrm{O}\right)$ (Sigma-Aldrich) aqueous solutions were mixed, stirred, and added dropwise to the oxidized-CNT to the point of incipient wetness. The catalyst was then dried for $3 \mathrm{~h}$ under a flowing air oven at $120{ }^{\circ} \mathrm{C}$ (the last drying step lasted for $6 \mathrm{~h}$ ), and the impregnation process was repeated until all the aqueous solutions of cobalt and iron nitrates were added to oxidizedCNT. The metal loading of the catalyst was determined by knowing the mass of the solution added during the impregnation of the catalysts.

The samples prepared using this colloidal synthesis (cs) will be given the following assignments: $5 \% \mathrm{cs}-\mathrm{Fe} / \mathrm{CNT}, 10 \%$ (cs$\left.\mathrm{Co}_{10} \mathrm{Fe}_{90} / \mathrm{CNT}\right), 10 \%\left(\mathrm{cs}-\mathrm{Co}_{30} \mathrm{Fe}_{70} / \mathrm{CNT}\right), 10 \%\left(\mathrm{cs}^{-} \mathrm{Co}_{50} \mathrm{Fe}_{50} /\right.$ $\mathrm{CNT}$ ), $10 \%$ (cs-Co $\mathrm{Co}_{70} \mathrm{Fe}_{30} / \mathrm{CNT}$ ), and $5 \% \mathrm{cs}-\mathrm{Co} / \mathrm{CNT}$ while those prepared by incipient wetness coimpregnation (ci) in the catalytic testing section will be represented as $5 \%$ (ci-Co/ $\mathrm{CNT})$ and $10 \%\left(\mathrm{ci}-\mathrm{Co}_{50} \mathrm{Fe}_{50} / \mathrm{CNT}\right)$. Where the percentage in the beginning of the designations refers to the metal loading percentage by weight, and the subscript number next to each metal refers to its percentage in the assigned metal loading. For example, $10 \%\left(\mathrm{Co}_{50} \mathrm{Fe}_{50}\right) / \mathrm{CNT}$ means that the metal loading is
$10 \%$ by weight of the whole catalyst and that Co loading is $5 \%$ by weight and $\mathrm{Fe}$ is $5 \%$ by weight.

Inductively Coupled Plasma-Mass Spectroscopy (ICP-MS). The freshly prepared samples were characterized by inductively coupled plasma (ICP) mass spectrometry at Kolbe laboratories, Germany, to verify the metal loading and cobalt and iron percentages in the catalyst.

X-ray Powder Diffraction (XRD). X-ray powder diffraction (XRD) measurements were conducted on the freshly calcined and the spent catalysts using a Bruker D2 Phaser diffractometer with $\mathrm{Co} K \alpha_{1,2}$ radiation $(\lambda=1.789 \AA)$ to study the formed chemical phases on the catalysts after preparation and after catalysis. Crystallite size estimation was carried out using the Debye-Scherrer equation implemented in the DiffracEvaluation software provided by Bruker.

In situ XRD experiments were carried out using the Bruker D8 Advance X-ray diffractometer and Co $\mathrm{K} \alpha_{1,2}(\lambda=1.790 \AA)$ radiation. The diffractometer was equipped with an AntonPaar XRK900 reaction chamber. The fresh sample $(40 \mathrm{mg}$ ) was heated up to $350{ }^{\circ} \mathrm{C}$ with a ramp of $3{ }^{\circ} \mathrm{C} \cdot \mathrm{min}^{-1}$ under Helium atmosphere. After reaching $350{ }^{\circ} \mathrm{C}$, reduction of the samples was initiated by switching the gas inlets to a mixture of $\mathrm{He} / \mathrm{H}_{2}(2: 1)$ for $2 \mathrm{~h}$. XRD measurements were conducted between $35^{\circ}<2 \theta<80^{\circ}$ every $0.14^{\circ}$ with $3 \mathrm{~s} \cdot$ step $^{-1}$. Background subtraction was conducted by DiffracEvaluation software, and the data was plotted in Origin.

Transmission Electron Microscopy. The freshly prepared catalysts were characterized by transmission electron microscopy (TEM). Specimens were prepared by suspending them in a small amount of ethanol followed by ultrasonic bath to improve specimen dispersion. The samples were then dropped on copper grids with holey carbon film. The samples were analyzed using Tecnai 12 and Tecnai 20 microscopes with electron beam voltages of 120 and $200 \mathrm{keV}$, respectively. The spent catalysts were characterized by scanning transmission electron microscopy-high-angle annular dark field (STEM-HAADF) images using an FEI Talos F200X transmission electron microscope with electron beam voltages of $200 \mathrm{keV}$.

Image analysis was carried out using ImageJ and Gatan Digital Micrograph. Particle size analysis was conducted automatically on 2000 nanocrystals using ImageJ and manually on 300 supported nanoparticles.

Energy Dispersive X-ray Spectrometry (EDX) and Electron Energy Loss Spectroscopy (EELS). The fresh and spent catalysts were characterized using an energy-dispersive $\mathrm{X}$-ray spectrometer (EDX) in the Tecnai 20F and FEI Talos F200X microscopes using an electron beam voltage of 200 $\mathrm{keV}$. EDX can give information on the elemental composition of the catalyst as well as the elemental concentration of each sample. The concentration quantification step was carried out after removing the spectral background using the Tecnai Imaging and Analysis (TIA) software.

The high-resolution scanning transmission electron microscopy combined with electron energy loss spectroscopy (STEM-EELS) measurements were conducted on the fresh and reduced samples. The fresh samples were reduced in a confined environment under hydrogen atmosphere and then rapidly transferred into the microscope chamber with limited exposure to air. The samples to be measured were sonicated in ethanol and dropped onto a holey amorphous carbon film supported on a copper grid. 

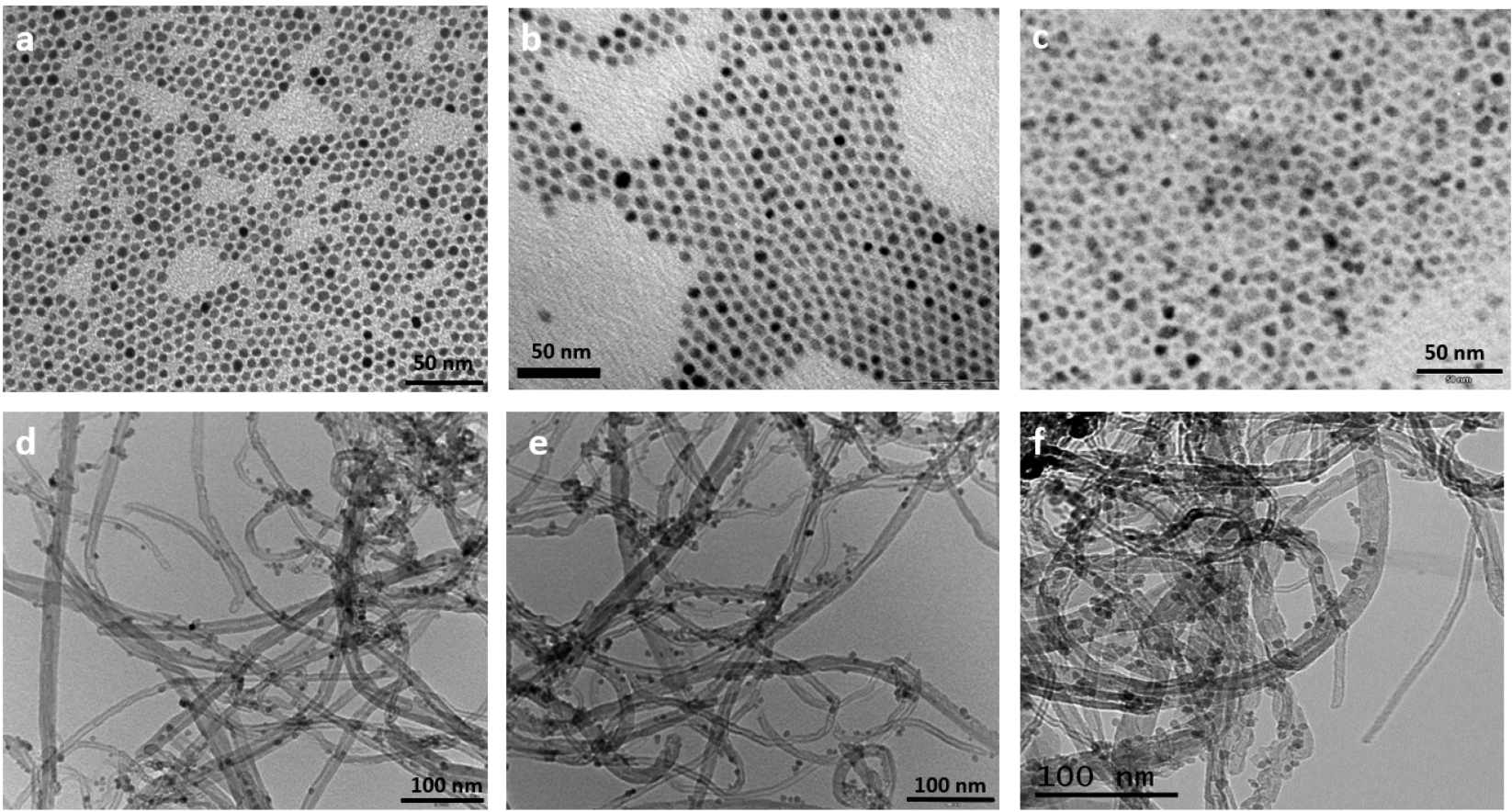

Figure 1. Transmission electron microscopy (TEM) images of cobalt-iron oxide bimetallic nanocrystals with different ratios after synthesis (top) and after being supported on CNT (bottom). The metal loading of all catalysts is $10 \mathrm{wt} \%$, and the cobalt iron ratios in different catalysts are the following: (a) bimetallic nanocrystals of $\mathrm{Co}_{30} \mathrm{Fe}_{70}$ oxides and (d) the same nanocrystals are supported on CNT. (b) Shows the Co $\mathrm{Co}_{50} \mathrm{Fe}_{50}$ oxide nanocrystals and (e) is the $\mathrm{Co}_{50} \mathrm{Fe}_{50} / \mathrm{CNT}$ catalyst. (c, f) Show the $\mathrm{Co}_{70} \mathrm{Fe}_{30}$ oxide nanocrystals and the $\mathrm{Co}_{70} \mathrm{Fe}_{30} / \mathrm{CNT}$ catalyst respectively. The scale bar in $(\mathrm{a}-\mathrm{c})$ is $50 \mathrm{~nm}$, and the scale bar in $(\mathrm{d}-\mathrm{f})$ is $100 \mathrm{~nm}$.

Two electron microscopes were employed for measurements: The first is a VG Cold HB 501 microscope, which is equipped with a cold field emission gun (CFEG) and parallel Gatan 666 EELS capabilities optically coupled to a low-noise liquid-nitrogen-cooled CCD camera. The microscope can produce EELS spectra with a spectral resolution of $0.5 \mathrm{eV}$ and subnanometer spatial resolution. ${ }^{28}$ The other microscope employed during the STEM-EELS experiments was the aberration-corrected Nion UltraStem 200 microscope, which is also fitted with a CFEG and operated at $60-100 \mathrm{keV}$ and can provide atomically resolved images.

The spectroscopic information acquisition was carried out using the spectrum image acquisition mode. ${ }^{29}$ Spectral images were generated by assigning a region of interest (ROI) in the sample and then scanning the region pixel-by-pixel while acquiring a whole EELS spectrum for each pixel. Some preventive measures have been taken to minimize contamination during observations by heating the sample under vacuum at $100{ }^{\circ} \mathrm{C}$ for a couple of hours before introduction into the microscope column. In addition, samples were put under high vacuum conditions in the microscope column $(\sim 1.8 \mathrm{mbar})$ to further minimize the contamination. The samples were studied using two different imaging modes: the high-angle annular dark field mode (HAADF) and the bright field mode (BF).

The data were analyzed using the Gatan Digital Micrograph software. EELS elemental maps were generated for certain areas in each sample to allow studying the spatial distribution of cobalt and iron in the calcined and reduced samples. Crosssectional estimation and elemental quantitative analysis were conducted using the Gatan EELS Analysis tool.

Catalytic Testing at High-Temperature FT Conditions. The Fischer-Tropsch Synthesis in the high-temper- ature regime was carried out at 1 bar and $350^{\circ} \mathrm{C}$ in a plug flow reactor. Twenty milligrams of the catalyst with a particle size ranging from 0.2 to $0.4 \mathrm{~mm}$ and bed density of $0.2 \mathrm{~g} \mathrm{~mL}^{-1}$ was diluted with around $200 \mathrm{mg}$ of silica carbide ( $\mathrm{SiC}$ ) with a particle size of $0.2 \mathrm{~mm}$. The diluted catalyst was then reduced in situ at $350{ }^{\circ} \mathrm{C}$ (with a heating ramp rate of $5{ }^{\circ} \mathrm{C} / \mathrm{min}$ ) under a flow $\mathrm{H}_{2} / \mathrm{Ar}$ (with a flow rate of $40 \mathrm{~mL} / \mathrm{min} \mathrm{Ar}$ and $20 \mathrm{~mL} /$ $\min \mathrm{H}_{2}$ ). The reduction process lasted for $2 \mathrm{~h}$.

The catalytic reaction was then initiated at the same temperature while changing the gases stream from $\mathrm{H}_{2} / \mathrm{Ar}$ to $\mathrm{H}_{2} / \mathrm{CO}$ mixture (with flow rate of $3 \mathrm{~mL} / \mathrm{min}$ for each gas). The products composition up to $\mathrm{C}_{16}$ carbon chains was analyzed using gas chromatography equipped with a Varian CP3800 analyzer and a flame ionization detector FID (Column CP Sil $5 \mathrm{CB}$ ) to determine the activity, selectivity, and stability of the catalyst under study after $19 \mathrm{~h}$ of FT at low CO conversion $(0.5-1 \%)$. The catalyst selectivity for every set of products related to a specific carbon atom number was calculated by comparing the amount of carbon atoms in a product relative to the amount of total carbon atoms present in the all hydrocarbons produced. We would like to note that although iron-based catalyst shows high activity and selectivity toward the water-gas shift (WGS) reaction at HTFT conditions, we did not investigate this reaction because it is outside the scope of this study. Interested readers are kindly referred to in depth studies on the WGS on cobalt-iron bimetallic catalysts. $^{33,58}$

Catalytic Testing at Low-Temperature FT Conditions. The Fischer-Tropsch Synthesis in the low-temperature regime was conducted similarly to the high-temperature FT but with a change in the temperature program and gases concentrations. Twenty milligrams of the catalyst (with particle sizes of $0.2-$ $0.4 \mathrm{~mm})$ was diluted by $\mathrm{SiC}(0.2 \mathrm{~mm})$ and loaded into a plug 
Table 1. Shows the Elemental Loadings by Weight Percent of Iron and Cobalt Using ICP (with Respect to the Whole Sample and with Respect to Each Element) and by $\mathrm{EDX}^{a}$

\begin{tabular}{|c|c|c|c|c|c|c|c|c|c|}
\hline \multirow[b]{3}{*}{ samples } & \multicolumn{2}{|c|}{ ICP } & \multicolumn{2}{|c|}{ ICP } & \multicolumn{2}{|c|}{ EDX } & \multirow[b]{3}{*}{$\begin{array}{l}\text { particle size } \\
(\mathrm{nm})\end{array}$} & \multirow[b]{3}{*}{$\begin{array}{c}\text { ICP } \\
\text { uncertainty } \\
(\%)\end{array}$} & \multirow[b]{3}{*}{$\begin{array}{c}\text { EDX } \\
\text { uncertainty } \\
(\%)\end{array}$} \\
\hline & \multicolumn{2}{|c|}{$\begin{array}{l}\text { loading by weight } \% \text { relative to } \\
\text { the whole sample }\end{array}$} & \multicolumn{2}{|c|}{$\begin{array}{c}\text { loading by weight } \% \text { relative to } \\
\text { each other }\end{array}$} & \multicolumn{2}{|c|}{$\begin{array}{c}\text { loading by weight } \% \text { relative to } \\
\text { each other }\end{array}$} & & & \\
\hline & $\begin{array}{l}\text { iron loading } \\
\text { (wt \%) }\end{array}$ & $\begin{array}{l}\text { cobalt loading } \\
\text { (wt \%) }\end{array}$ & $\begin{array}{l}\text { iron loading } \\
\text { (wt \%) }\end{array}$ & $\begin{array}{l}\text { cobalt loading } \\
\text { (wt \%) }\end{array}$ & $\begin{array}{l}\text { iron loading } \\
\text { (wt \%) }\end{array}$ & $\begin{array}{l}\text { cobalt loading } \\
\text { (wt \%) }\end{array}$ & & & \\
\hline $\mathrm{Co}_{30} \mathrm{Fe}_{70} / \mathrm{CNT}$ & 7.6 & 3.5 & 68.5 & 31.5 & 71 & 29 & 5.2 & 0.01 & 2 \\
\hline $\mathrm{Co}_{50} \mathrm{Fe}_{50} / \mathrm{CNT}$ & 5.3 & 5.4 & 49.6 & 50.4 & 51 & 49 & 4.9 & 0.03 & 1.5 \\
\hline $\mathrm{Co}_{70} \mathrm{Fe}_{30} / \mathrm{CNT}$ & - & - & - & - & 25 & 75 & 5.7 & - & 5 \\
\hline $\mathrm{Co}_{10} \mathrm{Fe}_{90} / \mathrm{CNT}$ & - & - & - & - & 87 & 13 & 7.9 & - & 4 \\
\hline $\mathrm{Fe}_{100} / \mathrm{CNT}$ & - & - & - & - & 100 & 0 & 5 & - & 1.5 \\
\hline cs_Co ${ }_{100} / \mathrm{CNT}$ & - & - & - & - & 0 & 100 & 6 & - & 1.5 \\
\hline ci_Co $\mathrm{Co}_{100} / \mathrm{CNT}$ & - & - & - & - & 0 & 100 & 8 & - & 1.5 \\
\hline
\end{tabular}

${ }^{a}$ The average particle size was measured by a particle size distribution analysis of 300 nanoparticles from TEM images.
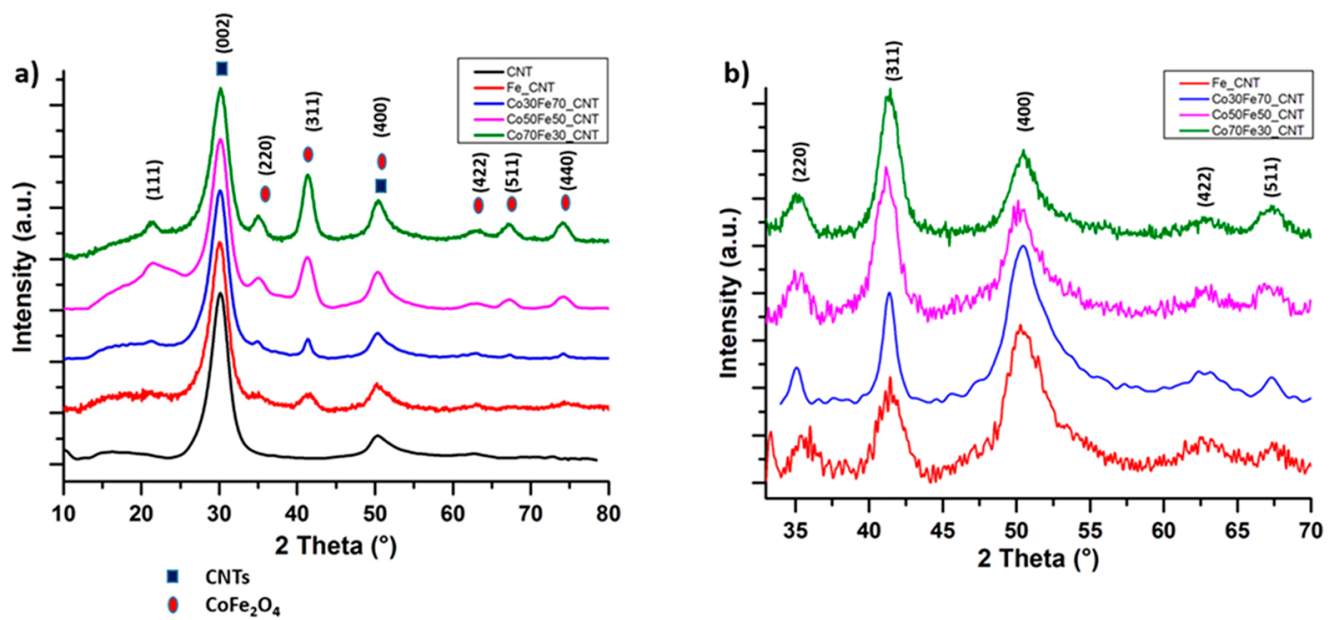

Figure 2. (a) X-ray diffraction patterns of CNT-supported cobalt and iron oxide catalysts with different concentrations of iron and cobalt. (b) A close up of the XRD pattern from $2 \theta 35^{\circ}-70^{\circ}$.

flow reactor. The catalyst was then reduced for $2 \mathrm{~h}$ at $350{ }^{\circ} \mathrm{C}$ (heating ramp rate of $5{ }^{\circ} \mathrm{C} / \mathrm{min}$ ) under a flow $\mathrm{H}_{2} / \mathrm{Ar}$ (with a flow rate of $1: 3 \mathrm{v} / \mathrm{v}$ ). After reduction, the temperature was lowered to $220{ }^{\circ} \mathrm{C}$, and the gas mixture was changed to $\mathrm{H}_{2} /$ $\mathrm{CO}$ (flow rate of $6 \mathrm{~mL} / \mathrm{min}$ for $\mathrm{H}_{2}$ and $3 \mathrm{~mL} / \mathrm{min}$ for $\mathrm{CO}$ ). The catalytic reaction was performed for $40 \mathrm{~h}$, and the produced hydrocarbons were analyzed every hour using online gas chromatography (Varian 430-GC instrument).

The catalyst time yield or the amount of $\mathrm{CO}$ converted per unit mass of metal (M) catalyst per second is given by

$$
\text { catalyst time yield }=\frac{\text { moles of CO converted }}{m_{\mathrm{M}} \times \text { time }}
$$

The same concept can be used in the case of iron, where the only change is the use of the unit mass of iron:

$$
\mathrm{FTY}=\frac{\text { moles of CO converted }}{m_{\mathrm{Fe}} \times \text { time }}
$$

The catalyst selectivity based on hydrocarbons only at ambient pressure can be calculated from the following equation:

$$
S_{\mathrm{C}_{n}}(C \%)=\frac{n \times C_{\mathrm{C}_{n}}}{\sum_{m} m \times C_{\mathrm{C}_{m}}} \times 100 \%
$$

and the selectivity of $\mathrm{C}_{5+}$ hydrocarbons (up to $\mathrm{C}_{18}$ ):

$$
S_{\mathrm{C}_{5+}}(C \%)=\sum_{n=5}^{18} S_{\mathrm{C}_{n}}
$$

\section{RESULTS AND DISCUSSION}

Structure and Composition of Fresh Catalysts. In the first step of the catalyst fabrication, spinel cobalt iron oxide nanocrystals with different cobalt/iron ratios and particle sizes of around 5-7 nm were synthesized using nonhydrolytic colloidal synthesis adapted from the work of Sun et al. ${ }^{25}$ The cobalt to iron ratio was controlled by changing the concentrations of cobalt precursor used in the synthesis process with respect to iron precursor concentration. This synthesis method provides not only a full control over the ratio of the different elements in the nanocrystals, but also a control over the nanocrystal size by varying the volume of the injected precursor and the reflux temperature. ${ }^{25,26}$

The cobalt iron oxide nanocrystals were then supported on carbon nanotubes (CNT) by mixing them in the presence of octadecene solvent under nitrogen atmosphere and heating at $200{ }^{\circ} \mathrm{C}$ for $30 \mathrm{~min}$. It was found by Casavola et al. that this heating time is the optimum for acquiring a catalyst with homogeneous distribution of the nanocrystals on the CNT. ${ }^{27}$ They also found that the experiment temperature achieves the thermodynamic equilibrium at which the nanocrystals can be 
homogeneously dispersed on the external surface of the support. Using this method, we have prepared a catalyst with 10 wt \% metal loading of cobalt and iron by controlling the volume and the weight of the cobalt iron oxide nanocrystals and the CNT support, respectively.

The average particles size distribution of the supported nanoparticles was determined by statistical analysis of their TEM micrographs over about 300 nanocrystals (Figure 1 and Table 1). The cobalt-to-iron ratios and their contribution to the total sample metal loading were confirmed by ICP-MS and EDX. This particle size analysis of the supported bimetallic nanoparticles showed that the nanoparticles' size did not change dramatically after the CNT-supporting procedure and after removing the organic surfactants (see Figures S2 and S3), which demonstrates the high stability of the bimetallic nanoparticles and the role of CNT as ligands that attach to the surface of nanocrystals by a partial electron-donor effect. ${ }^{30}$ This interaction reduces the surface free energy of the oxide nanocrystals and allows them to stabilize on the CNT. Finally, the introduction of a catalyst calcination step (heating at 300 ${ }^{\circ} \mathrm{C}$ under inert atmosphere) after the catalyst drying procedure did not show dramatic enhancement of the nanoparticles distribution over the support.

X-ray diffraction (XRD) characterization of the catalysts provides valuable information on the catalysts' crystal structure and crystallites' size. XRD analysis of the CNT, monometallic iron catalyst, and the bimetallic catalysts showed expected broad lines in all diffractograms because of the small size of the crystallites (Figure 2). The (002) and (004) diffraction peaks of CNT at $29.8^{\circ}$ and $51^{\circ}$ indicate the presence of graphite and show that CNT retained the crystal structure after metal deposition. ${ }^{31}$ The iron oxide monometallic catalyst was found to possibly have a maghemite structure $\left(\gamma-\mathrm{Fe}_{2} \mathrm{O}_{3}\right),{ }^{27}$ while the bimetallic catalysts contained a cobalt ferrite inverse spinel structure $\left(\mathrm{CoFe}_{2} \mathrm{O}_{4}\right) \cdot{ }^{26,32-34}$ By examining the relative intensity of the (311) and (400) diffraction lines at $2 \theta$ values of $42.5^{\circ}$ and $50.5^{\circ}$ for all four catalysts (Figure $2 \mathrm{~b}$ ), we noticed that the relative intensity of these two diffraction lines is reversed when the cobalt content in the catalyst was equal to or higher than the iron content in the catalyst. For example, in the $\mathrm{Fe} / \mathrm{CNT}$ catalyst, the fwhm of the (311) diffraction line is 1.6062 and that for (400) diffraction line is 3.203. While for the $\mathrm{Co}_{70} \mathrm{Fe}_{30} / \mathrm{CNT}$ catalyst, the fwhm of the (311) diffraction line is 1.5501 and that for (400) diffraction line is 2.3234. This may hint to the relation between increasing the cobalt content and the enhancement of crystallinity of the catalysts across the 311 orientation.

In order to get a more precise insight on the structure and chemical composition of cobalt iron bimetallic catalysts, studying the elemental composition on the nanoparticle scale is necessary. Therefore, we performed high-resolution STEMEELS analysis of the composition of the fresh catalysts with different cobalt-iron ratios. In this section, we will discuss the STEM-EELS analysis of the $10 \%\left(\mathrm{Co}_{50} \mathrm{Fe}_{50} / \mathrm{CNT}\right)$ catalyst (Figure 3a). The STEM-EELS analysis of catalysts with other $\mathrm{Co}-\mathrm{Fe}$ ratios and more details on the metal ratios quantification process can be found in the Supporting Information. Analysis of the fresh $\mathrm{Co}_{50} \mathrm{Fe}_{50} / \mathrm{CNT}$ sample confirms the cubic structure of the $5 \mathrm{~nm}$ nanoparticle. 2D EELS elemental maps were generated by selectively choosing the cobalt and iron $\mathrm{L}_{2,3}$-edge energy (and K-edge for oxygen) and filtering out all the edge energies of other elements in the sample after background subtraction. Principal component

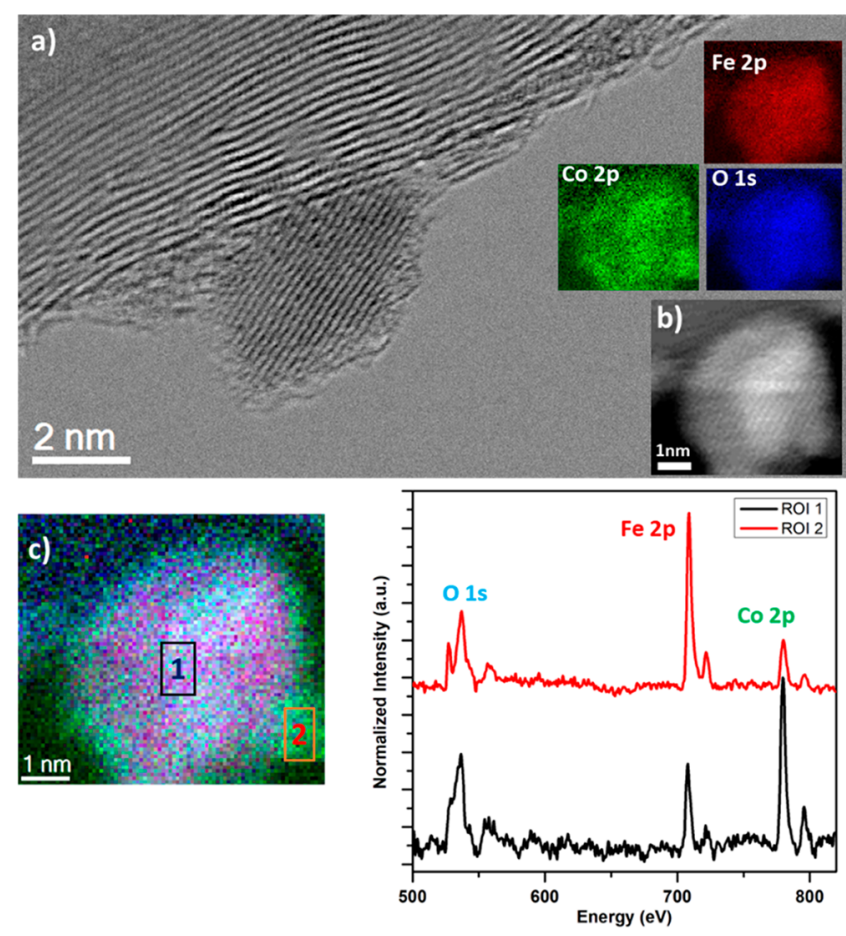

Figure 3. (a) Bright field TEM image that shows the $5 \mathrm{~nm}$ cobalt iron bimetallic nanoparticle (50:50) supported on CNT. (b) Spectral image of the CNT-supported nanoparticle combined with the elemental maps of each species in the nanoparticle. The cobalt, iron, and oxygen maps were generated by filtering their EELS edges in the spectra. (c) An RGB composite of iron (red), cobalt (green), and oxygen (blue), with a selective EELS spectrum generated from the center and outer edge regions in the nanoparticle.

analysis (PCA) was then carried out to denoise the generated maps by removing the eigen functions that contained the background noise. ${ }^{35,36}$ Analysis of the 2D EELS maps showed that cobalt, iron, and oxygen atoms were generally homogeneously distributed over the nanoparticle, which confirmed the presence of both cobalt and iron in one spinel structure. Investigation of other supported nanoparticles in different regions of the fresh catalyst confirmed that all the nanoparticles retained their bimetallic character after attachment on the CNT support (Figure S5).

Further analysis of the EELS elemental maps showed that although all the three elements were present homogeneously over the bimetallic supported nanoparticle, there was a relative increase in the concentration of cobalt at the outer layer of the nanoparticle, while iron had a preferential distribution in the nanoparticle core as can be observed be comparing the relative intensities of $\mathrm{Co}$ and $\mathrm{Fe} 2 \mathrm{p}$ EELS spectra in two different regions (Figure $3 \mathrm{~b}$ ). This behavior was also observed consistently after analyzing other nanoparticles in the fresh $\mathrm{Co}_{50} \mathrm{Fe}_{50} / \mathrm{CNT}$ catalyst (Figure S5).

Catalyst Reducibility. The reduction behavior of the $\mathrm{Co}_{50} \mathrm{Fe}_{50} / \mathrm{CNT}$ catalyst was characterized using in situ XRD where the sample was first investigated during heating in an inert atmosphere calcination at $350{ }^{\circ} \mathrm{C}$ (heating ramp $10{ }^{\circ} \mathrm{C} /$ $\min )$ then upon reduction using a mixture of $\mathrm{He} / \mathrm{H}_{2}(1: 1)$ for 2 hours (Figure 4). During heating in an inert atmosphere, the cobalt ferrite spinel structure was found to be the prevailing phase. However, upon switching to the reduction mixture, the sample was directly reduced to a cubic iron monoxide $(F m \overline{3} m)$ 


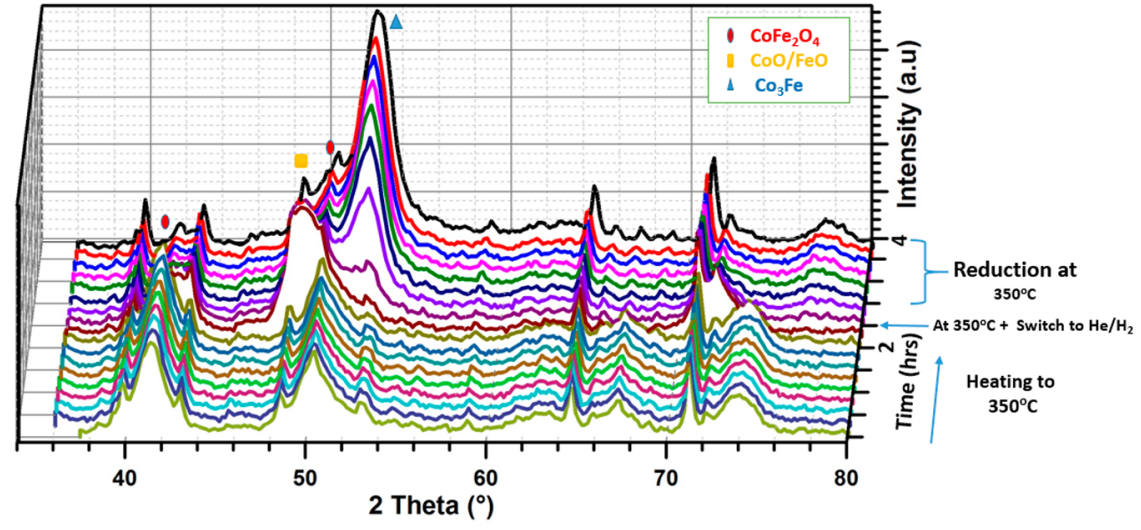

Figure 4. In situ X-ray diffraction pattern of the reduction process of $10 \%\left(\mathrm{Co}_{50} \mathrm{Fe}_{50} / \mathrm{CNT}\right)$ catalyst.
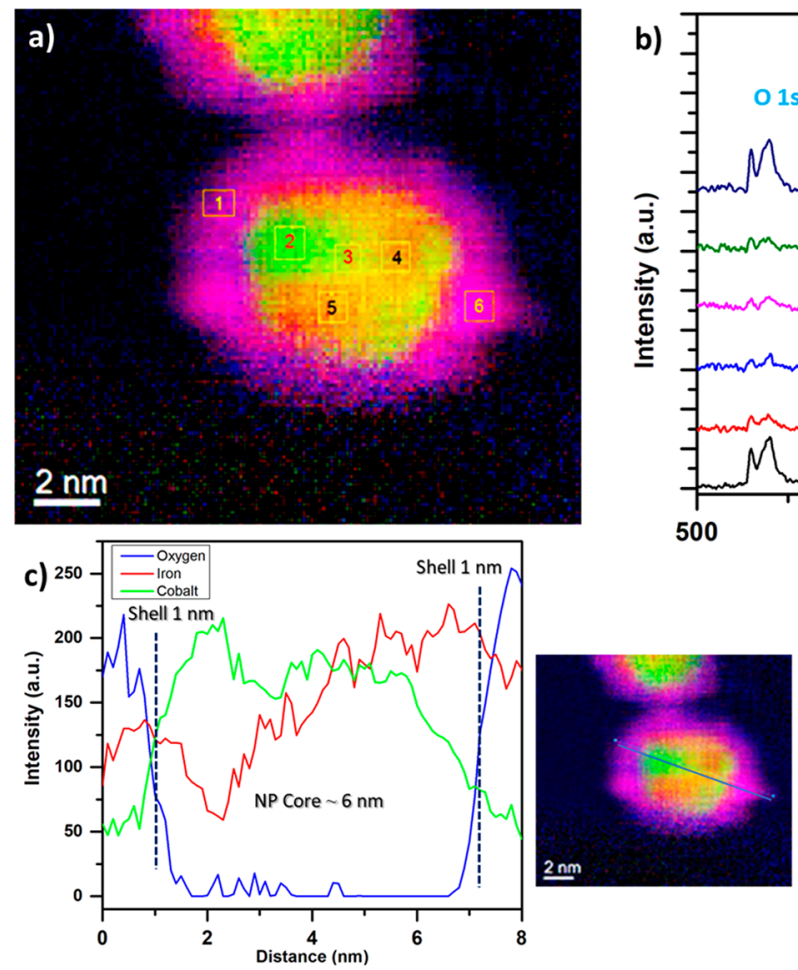

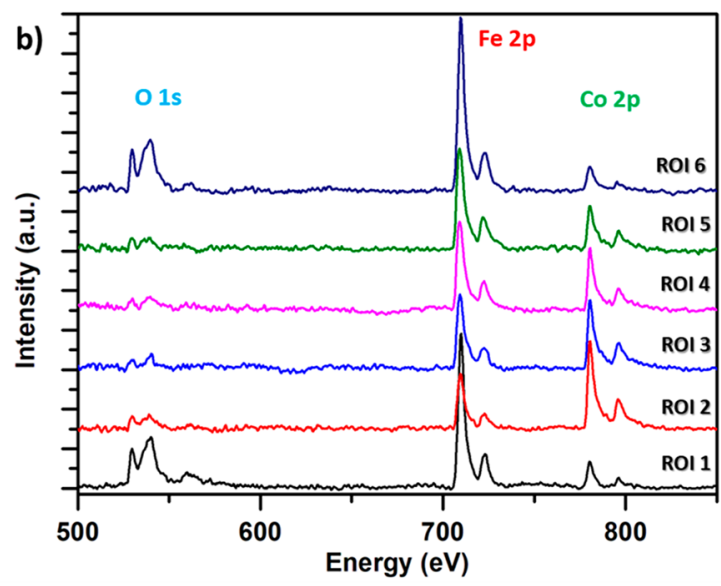

d)

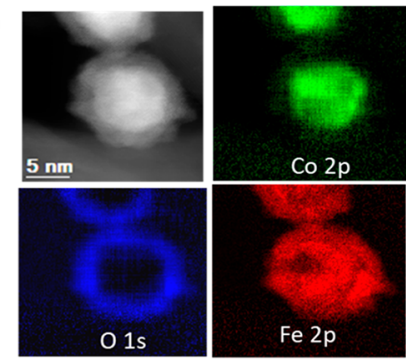

Figure 5. (a) An RGB composite of iron (red), cobalt (green), and oxygen (blue) species in the reduced nanoparticle. (b) EELS spectra extracted for each region of interest (ROI) in the nanoparticle (represented by number in the RGB). (c) A line profile drawn on the same RGB composite to show the concentration distribution of the three elements in the particle. (d) HAADF image of the CNT-supported nanoparticle and the generated elemental maps of $\mathrm{Co}, \mathrm{Fe}$, and $\mathrm{O}$.

and cobalt monoxide with reference to PDF database number (00-003-0968) and (00-048-1719), respectively (note the broad peak at $\left.49^{\circ}\right)$. These short-lived $(25 \mathrm{~min})$ mixed monoxide phases soon started to further reduce into the cobalt-iron metallic phase (PDF 00-048-1816). By increasing the reduction time, it became clear that the formation of metallic cobalt and iron increases as could be noted from the increased intensity of the metallic patterns (and disappearance of the broad monoxides peaks). However, by the end of the reduction process, it was not clear whether all the cobalt and iron were fully reduced to their metallic states. Furthermore, we only noticed the appearance of one cobalt iron metallic phase represented by mainly one intense diffraction line at $53^{\circ}$, but we did not observe any other diffraction lines representative to any other metallic phase solely specific to cobalt or to iron. This is in agreement with the XRD study of
CoFe/CNF by Díaz et al. ${ }^{13}$ and in contrast with other XRD studies on $\mathrm{CoFe} / \mathrm{SiO}_{2}{ }^{37,38}$ This difference offers and indication on the role of the metal oxide supports and the different cobalt-iron ratios in dictating the reduction behavior of the catalyst. ${ }^{39}$ Finally, previous studies proposed that the $\mathrm{Co}-\mathrm{Fe}$ metallic diffraction line might be attributed to the presence of a $\mathrm{Co}-\mathrm{Fe}$ alloy phase, ${ }^{13,40-42}$ but we believe that depending only on this in situ XRD study cannot be conclusive in confirming this assumption.

High-resolution STEM-EELS analysis of the reduced $\mathrm{Co}_{50} \mathrm{Fe}_{50} / \mathrm{CNT}$ nanoparticles showed that cobalt and iron distribution in the nanoparticle changed dramatically upon reduction (Figure 5a). First, the reduced nanoparticle was found to have a core-shell structure, where the core $(5-6 \mathrm{~nm})$ contains cobalt and iron in their metallic states and the shell is mainly an oxide layer. This oxide layer $(1-1.5 \mathrm{~nm})$ was formed 
Table 2. Catalytic Testing Results at HTFT at 1 bar, $350^{\circ} \mathrm{C}, \mathrm{GHSV}=1900 \mathrm{~h}^{-1}, \mathrm{H}_{2} / \mathrm{CO}=3 / 3(\mathrm{v} / \mathrm{v})$

\begin{tabular}{|c|c|c|c|c|c|c|}
\hline \multirow[b]{2}{*}{ sample } & \multirow[b]{2}{*}{ CO conv. (\%) } & \multirow[b]{2}{*}{ FTY $\left(10^{-5} \mathrm{~mol}_{\mathrm{co}} / \mathrm{g}_{\mathrm{Fe}} \cdot \mathrm{s}\right)$} & \multicolumn{4}{|c|}{ selectivity (\%) } \\
\hline & & & $\mathrm{CH}_{4}$ & $\mathrm{C}_{2}-\mathrm{C}_{4}$ olefins & $\mathrm{C}_{2}-\mathrm{C}_{4}$ paraffins & $\mathrm{C}_{5+}$ \\
\hline $\mathrm{ci}-\mathrm{Co}^{50} \mathrm{Fe}^{50} / \mathrm{CNT}$ & 0.40 & 0.98 & 36 & 48 & 2 & 14 \\
\hline $\mathrm{cs}-\mathrm{Co}^{50} \mathrm{Fe}^{50} / \mathrm{CNT}$ & 0.55 & 1.20 & 34 & 48.8 & 2.2 & 15 \\
\hline $\mathrm{Fe}^{100} / \mathrm{CNT}$ & 0.21 & 0.76 & 32 & 57 & 3 & 8 \\
\hline
\end{tabular}

due to a brief air exposure during the introduction of the sample to the electron microscope right before imaging. The elemental maps, EELS spectra, and EELS line profiles showed that the outer layer consisted mainly of iron oxide (Figure 5 ROI 1 and ROI 6). The generated oxygen EELS map also showed that there is no oxygen found in the core of the nanoparticle. This iron oxide layer formation was consistently observed in eight different spots in the sample under imaging. This observation may suggest that upon a brief exposure to oxygen, some iron atoms have enriched the nanoparticles surface by interacting with oxygen to form iron oxide.

By further analyzing the elemental chemical maps and correlating them with different local spectra in the red-greenblue (RGB) image, we could identify the formation of phase separation between the reduced iron and cobalt species in the nanoparticles core (ROIs 2-5). We observed that nanoparticle core was divided into regions with higher cobalt metal concentration (as can be observed in ROI 2 and the cobalt peak concentration in the correlated EELS spectra) and higher concentration of iron metal (ROIs 4 and 5 and correlated EELS spectra), with region 3 marking an interface where both cobalt and iron concentrations are almost equal. A line profile spectrum was drawn along the nanoparticle and confirmed the above-mentioned observations (Figure 5c). The line profile, along with other TEM analysis showed that the average particle size of the reduced nanoparticles has increased from around 5 to $7 \mathrm{~nm}$ (including the surrounding oxide layer). Furthermore, a quantitative analysis of the nanoparticles using the Hartree-Slater model ${ }^{43,44}$ showed that the cobalt-iron ratio at the interfacial region between the cobalt- and iron-rich regions is almost 1:1 (Figure S6, Supporting Information).

By combining the observations from in situ XRD and STEM-EELS experiments, we can confirm that upon reduction, cobalt-iron metallic phases were indeed formed. In addition, we can confirm on the basis of the above observations that, on the nanoparticle scale, cobalt and iron form an alloy structure upon reduction. However, the Co-Fe alloy in this case did not have the structure of an ordered alloy that was previously reported and that we expected but rather a Janus particle-like nanoalloy structure, where both cobalt and iron seemed to have been segregated into two different regions in the nanoparticle. This apparent segregation may have formed because of restructuring of the cobalt ferrite spinel upon reduction and the formation of mixed monoxide phases, which may have played a role in changing the thermodynamic stability of the $\mathrm{Co}-\mathrm{Fe}$ oxide structure. Other factors that can lead to the formation of Janus-like Co-Fe alloys may include the amount of surfactants and precursors added during the synthesis of catalysts. ${ }^{45}$ We also tried to verify whether a thin layer of carbon may have formed between the iron oxide shell and the $\mathrm{Co}-\mathrm{Fe}$ metallic core reported in a previous study on a similar system, ${ }^{46}$ but we could not find that layer. Our interpretation for this is that the $\mathrm{Co}(\mathrm{CO})_{3}$ precursor used in the previous study may have contributed to this upon its decomposition which may have led to the formation of that carbon layer before the subsequent deposition of iron oxide.

It is important to note that our interpretation of the STEMEELS observation of the reduced $\mathrm{Co}-\mathrm{Fe}$ nanoparticle is different from that of the previous work of Zhang et al., ${ }^{46}$ where they reported the formation of an ordered $\mathrm{Co}-\mathrm{Fe}$ alloy from comparing the lattice distances of the $\mathrm{Co}-\mathrm{Fe}$ core with that of $\mathrm{Co}-\mathrm{Fe}$ alloy. We believe that although the nanoparticles' reduction procedure was relatively similar, the difference in the synthesis methodology and the position of the nanoparticles inside or outside the carbon nanotubes may have led to different observations of the nanoparticles behavior upon reduction.

It is also worth noting that we attempted to further study the reduction behavior of the cobalt-iron bimetallic catalysts using temperature-programmed reduction (TPR). However, this turned out to be practically challenging for our systems since the remaining organic ligands around the nanoparticles decomposes at around $300{ }^{\circ} \mathrm{C}$, resulting in a gain in the hydrogen feed (a positive hydrogen peak) in a critical reduction phase transformation, which made the interpretation of the catalyst reduction profile from TPR a challenging task.

Catalytic Behavior. In order to understand the catalytic behavior of both iron and cobalt species in the bimetallic catalysts, we conducted two different Fischer-Tropsch synthesis experiments at different operation temperatures and different conversion rates. The first catalytic experiment was conducted at relatively high temperature (will be referred to as HTFT) and was aimed to target the catalytic performance of iron species in the catalyst. At these conditions, olefins are the preferred products (the process is often referred to as FischerTropsch to olefins (FTO)). The standard optimized procedure for conducting HTFT measurements on iron catalysts was described in more detail by Torres Galvis et al. ${ }^{47,48}$

The second experiment was aimed to understand the cobalt species performance during FT by conducting the catalytic testing at conditions that were suitable for the paraffins formation on cobalt-based catalysts and therefore will be referred to as low-temperature Fischer-Tropsch (LTFT). To gain a meaningful insight on the catalytic activity of the bimetallic $\mathrm{CoFe}$ catalysts prepared by colloidal synthesis (will be referred to in this section as $\mathrm{cs}^{-} \mathrm{Co}_{50} \mathrm{Fe}_{50} / \mathrm{CNT}$ ), we compared its catalytic performance to bimetallic $\mathrm{CoFe}$ catalyst made via incipient wetness coimpregnation method (will be referred to in this section as ci- $\mathrm{Co}_{50} \mathrm{Fe}_{50} / \mathrm{CNT}$ ). We also tested monometallic cobalt catalysts made by both colloidal synthesis and impregnation as well as monometallic colloidally synthesized cs-Fe/CNT catalysts, which is known for its selectivity to longer-chain hydrocarbons at low-temperature conditions. We would like to note here that we limited our catalytic testing on monometallic cobalt catalysts to lowtemperature conditions because cobalt is known for its extensive selectivity to methane formation at high-temperature conditions $\left(>300^{\circ} \mathrm{C}\right) .^{7,49}$ 

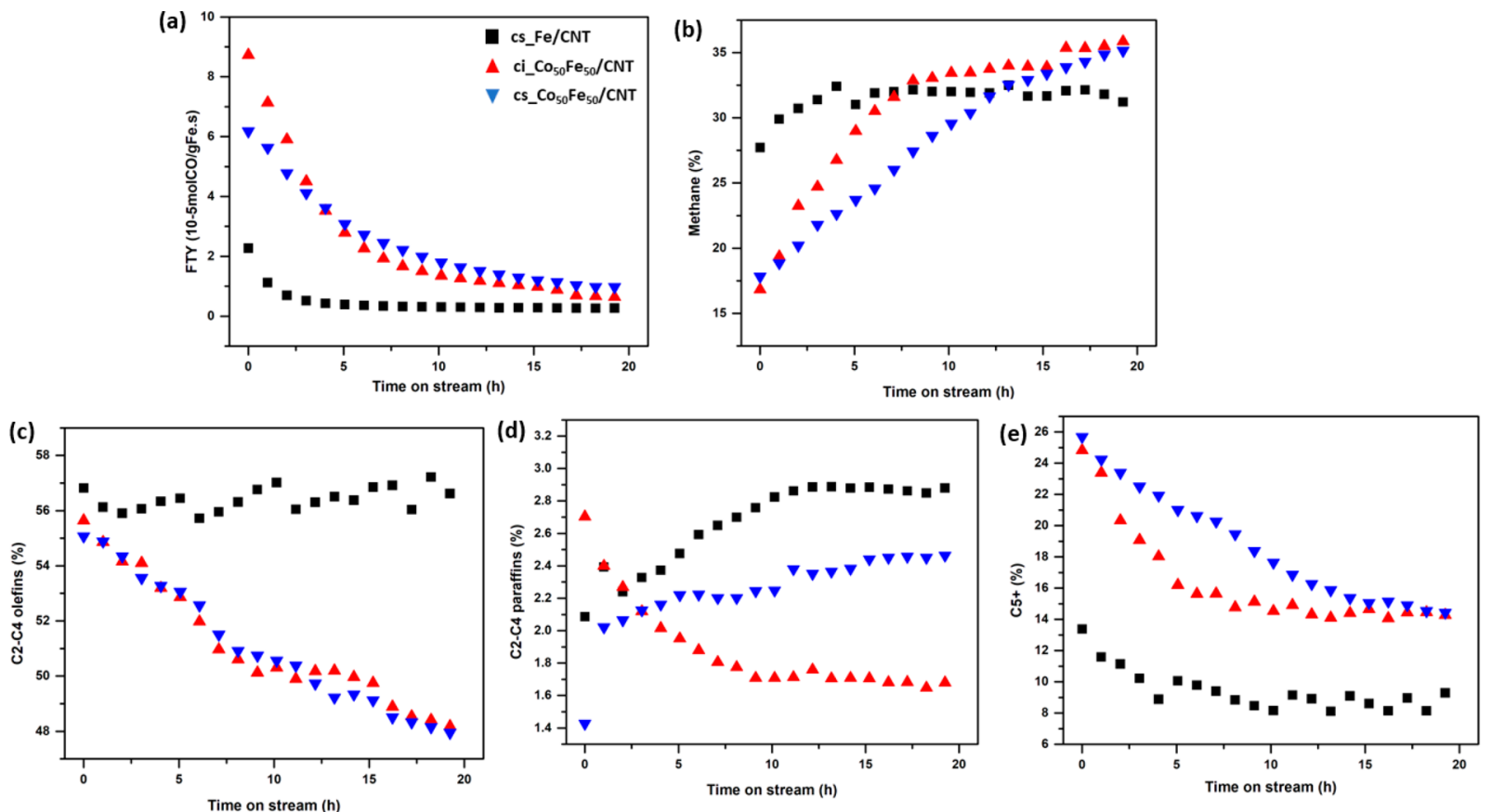

Figure 6. (a) Iron time yield (FTY) at $350{ }^{\circ} \mathrm{C}$ with time on stream for the monometallic $10 \%(\mathrm{Fe} / \mathrm{CNT}), 10 \%\left(\mathrm{cs}^{-} \mathrm{Co}_{50} \mathrm{Fe}_{50} / \mathrm{CNT}\right)$, and $10 \%(\mathrm{ci}-$ $\mathrm{Co}_{50} \mathrm{Fe}_{50} / \mathrm{CNT}$ ) catalyst. (b) Methane selectivity, (c) $\mathrm{C}_{2}-\mathrm{C}_{4}$ Olefins selectivity, (d) $\mathrm{C}_{2}-\mathrm{C}_{4}$ paraffins selectivity, and (e) $\mathrm{C}_{5+}$ products selectivity of the catalysts at HTFT conditions. The catalytic testing was conducted at $1 \mathrm{bar}, 350{ }^{\circ} \mathrm{C}$, GHSV $=1900 \mathrm{~h}^{-1}, \mathrm{H}_{2} / \mathrm{CO}=3 / 3$ (v/v). The catalyst names and symbols are the same in all four figures as in panel a.

During the HTFT, the iron time yield (FTY) and selectivity to olefins have been studied on the monometallic cs-Fe/CNT, bimetallic cs- $\mathrm{Co}_{50} \mathrm{Fe}_{50} / \mathrm{CNT}$, and $\mathrm{ci}-\mathrm{Co}_{50} \mathrm{Fe}_{50} / \mathrm{CNT}$ catalysts (Table 2). Upon reaching stabilization at $15 \mathrm{~h}$, it was observed that both the bimetallic catalysts had a considerably higher activity than the monometallic iron catalyst prepared by the same method. The noticeable decrease in the catalysts initial activity over time is similar to catalyst $5 \mathrm{AFeP}$ in ref 50 , which has a similar average particle size. This is probably due to either carbon deposition on the catalysts surface, the increase of particle size of iron, or structural changes that took place on the $\mathrm{Co}-\mathrm{Fe}$ metallic surfaces (Figure 6a). We believe that the last two explanations are more realistic because the magnitude of the catalyst deactivation in the first $10 \mathrm{~h}$ of reaction seems to fit more the change of cobalt and iron particle sizes and metallic structures that were already segregated in different regions in the nanoparticles as we previously observed. The methane selectivity of monometallic iron catalyst was found to be stable $(\sim 33 \%)$, while that of both bimetallic catalysts was rapidly increasing and did not seem to reach a stable plateau after $20 \mathrm{~h}$ on stream $(\sim 35 \%)$ (Figure $6 \mathrm{~b})$. On the other hand, the selectivity of the monometallic iron catalyst to $\mathrm{C}_{2}-\mathrm{C}_{4}$ olefins was found to be higher than the bimetallic catalyst (57\% vs $48 \%$ ) (Figure 6c) and had a comparable $\mathrm{C}_{2}-\mathrm{C}_{4}$ paraffins selectivity as well (Figure 6d). However, the bimetallic catalysts showed a considerably higher selectivity to $\mathrm{C}_{5+}$ hydrocarbons than the monometallic catalyst ( $57 \%$ vs 48\%) (Figure 6e). These observations show the strong role that the cobalt sites at the surface of the bimetallic catalysts play to direct the selectivity of the catalysts toward higher methane formation and lower olefins at HTFT conditions. ${ }^{49}$ We also found the results to be compatible with the catalytic testing observations of Arai et al., who observed that the $10 \%$ $\left(\mathrm{Co}_{50} \mathrm{Fe}_{50} / \mathrm{TiO}_{2}\right)$ catalyst prepared by impregnation gave the highest activity and selectivity toward $\mathrm{C}_{5+}$ hydrocarbons. ${ }^{51}$
Finally, the bimetallic $\mathrm{cs}^{-} \mathrm{Co}_{50} \mathrm{Fe}_{50} / \mathrm{CNT}$ and $\mathrm{ci}-\mathrm{Co}_{50} \mathrm{Fe}_{50} /$ CNT catalysts showed generally similar FTY activity and selectivity trends when compared to each other, which confirmed that the selectivity behavior of bimetallic cobaltiron catalysts in this study depends mainly on the catalysts composition rather than its synthesis method, with respect to the HTFT conditions.

On the other hand, testing the catalysts at LTFT conditions for $42 \mathrm{~h}$ under syngas stream showed that the catalytic activity of both bimetallic catalysts was almost identical (Table 3 and

Table 3. Catalytic Testing Results at HTFT at $1 \mathrm{bar}, 220^{\circ} \mathrm{C}$, $\mathrm{GHSV}=2000 \mathrm{~h}^{-1}, \mathrm{H}_{2} / \mathrm{CO}=6 / 3(\mathrm{v} / \mathrm{v})$

\begin{tabular}{|c|c|c|c|c|c|}
\hline \multirow[b]{2}{*}{ sample } & \multirow[b]{2}{*}{$\begin{array}{c}\mathrm{CO} \\
\text { conv. } \\
(\%)\end{array}$} & \multirow[b]{2}{*}{$\begin{array}{l}\text { catalyst time yield } \\
\left(10^{-5} \mathrm{~mol}_{\mathrm{CO}} / \mathrm{g}_{\mathrm{M}} \cdot \mathrm{s}\right)\end{array}$} & \multicolumn{3}{|c|}{ selectivity (\%) } \\
\hline & & & $\mathrm{CH}_{4}$ & $\begin{array}{c}\mathrm{C}_{2}- \\
\mathrm{C}_{4} \\
\text { olefins }\end{array}$ & $\mathrm{C}_{5+}$ \\
\hline $\mathrm{ci}-\mathrm{Co}^{50} \mathrm{Fe}^{50} / \mathrm{CNT}$ & 2.39 & 4.6 & 37 & 30 & 33 \\
\hline 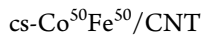 & 2.25 & 4.7 & 27 & 31 & 42 \\
\hline $\mathrm{Fe}^{100} / \mathrm{CNT}$ & 0.23 & 0.36 & 32 & 37.6 & 30.4 \\
\hline cs_Co ${ }^{100} / \mathrm{CNT}$ & 2.47 & 4.85 & 39.5 & 34.5 & 26 \\
\hline ci_Co ${ }^{100} / \mathrm{CNT}$ & 1.47 & 4.22 & 37 & 27 & 36 \\
\hline
\end{tabular}

Figure 7a). They both showed very high activity at the start of the reaction, possibly due to synergetic effects between cobalt and iron species in the catalyst. This activity decreased gradually to reach a semistable plateau after $\sim 15 \mathrm{~h}$. Comparing the bimetallic catalysts time yield with that of monometallic cobalt catalyst showed that the monometallic cobalt catalysts made by colloidal synthesis showed slightly higher activity when compared to bimetallic catalysts. It is intriguing, however, to note that the activity of the bimetallic catalysts is comparable to both monometallic cobalt catalysts made by different methods. This high activity could be attributed either 
(a)

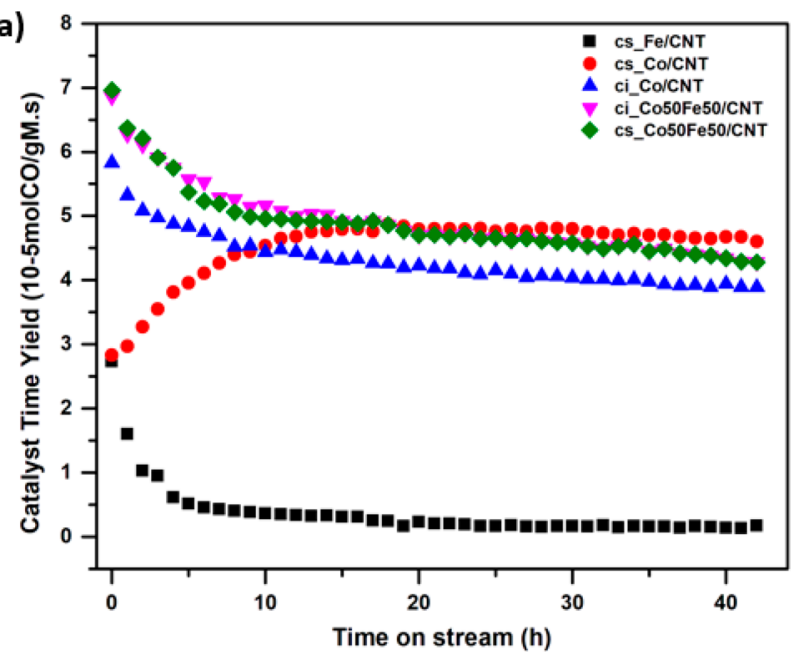

(c)

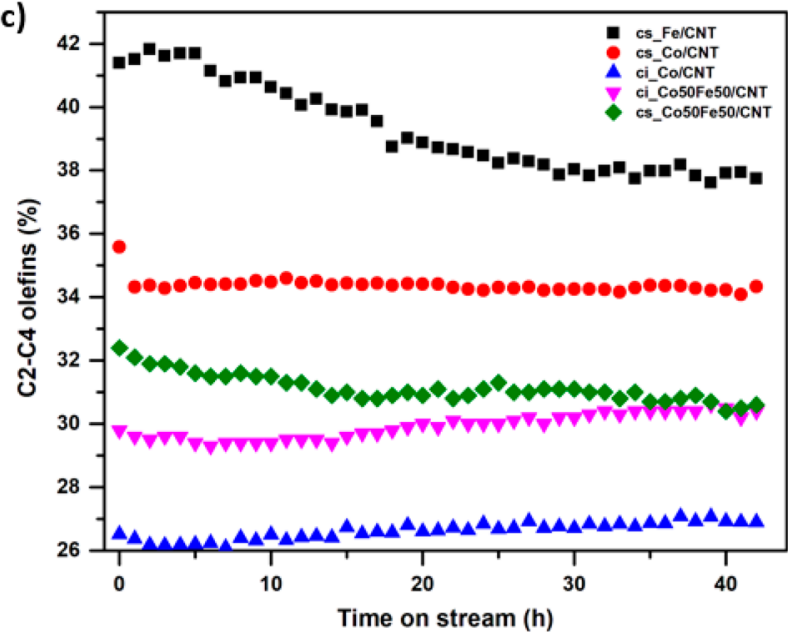

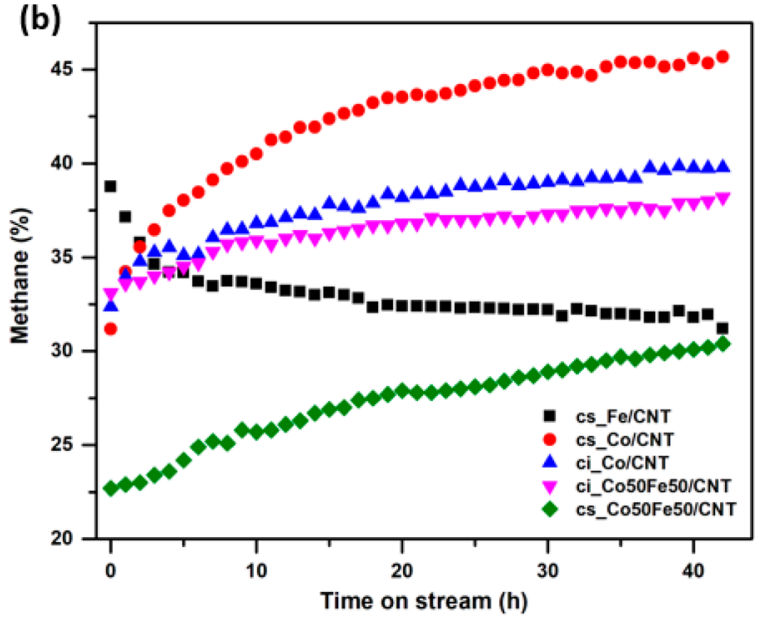

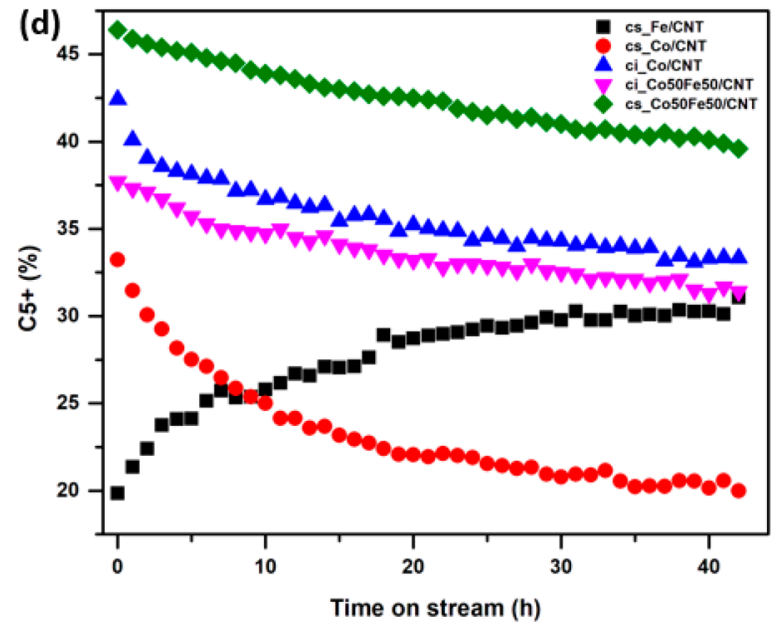

Figure 7. (a) Catalyst time yield at $220{ }^{\circ} \mathrm{C}$ with time on stream for the $10 \%\left(\mathrm{cs}_{-} \mathrm{Co}_{50} \mathrm{Fe}_{50} / \mathrm{CNT}\right.$ ), the $10 \%\left(\mathrm{ci}-\mathrm{Co}_{50} \mathrm{Fe}_{50} / \mathrm{CNT}\right.$ ) catalysts, and monometallic cobalt and iron catalysts. (b) Methane selectivity, (c) $\mathrm{C}_{2}-\mathrm{C}_{4}$ olefin selectivity, and (d) $\mathrm{C}_{5_{+}}$chain selectivity of the catalysts at LTFT conditions. The catalytic testing was conducted at 1 bar, $220{ }^{\circ} \mathrm{C}$, GHSV $=2000 \mathrm{~h}^{-1}, \mathrm{H}_{2} / \mathrm{CO}=6 / 3(\mathrm{v} / \mathrm{v})$.

to the enrichment of the catalysts surface by cobalt species at LTFT conditions or to the role that iron plays in enhancing the distribution of cobalt species over the support as we discussed earlier, which in turn, increases the catalyst surface area exposed to the syngas. This observation is in line with the observation reported on monometallic cobalt catalyst prepared with impregnation in ref 52 and 53, bearing in mind that the catalysts in these two studies were tested under 20 bar, while the bimetallic catalysts reported in our study were tested under 1 bar. We also believe that the better well-defined morphology and the better distribution of the cobalt nanoparticles in the colloidally synthesized cobalt catalysts is the reason for their relatively higher catalytic activity compared with impregnated cobalt catalysts. ${ }^{54}$ Another observation from the catalyst time yield of the colloidally synthesized cobalt catalysts was its gradual increase of activity in the first $10 \mathrm{~h}$ on stream. This behavior is different from that observed in the other tested catalysts which show a general decrease in activity in the first few hours on stream. We believe that the reason for this abnormal gradual increase in the activity of colloidally synthesized cobalt catalysts is the presence of residual carbonyl ligands in the catalysts after preparation. These ligands react with the hydrogen stream to form methane, which also explains the rapid increase in methane selectivity of this catalyst in the first $10 \mathrm{~h}$ on stream. Finally, iron monometallic catalysts show much lower catalytic time yield compared with cobalt and bimetallic catalysts, which is expected from iron at lowtemperature conditions.

On the other hand, the product selectivity behavior of the catalysts is puzzling. While the monometallic impregnated cobalt catalysts showed predictable products selectivity (high $\mathrm{C}_{5+}$ formation and low olefins selectivity), colloidally synthesized cobalt catalysts showed the opposite behavior. We are not sure of the origin of this selectivity preferences of the catalyst made with colloidal synthesis. However, we do note that in the case of the impregnated cobalt catalyst, the cobalt nanoparticles were attached on oxidized CNT support, while the colloidally synthesized cobalt nanoparticles were attached on "inert" CNT support. This oxidized support surface may have played a role in stabilizing the hydrocarbons adsorption on the catalyst surface and, hence, improving the longer chain hydrocarbons formation on the impregnated monometallic cobalt catalysts. ${ }^{52,55}$ In addition, high methane formation and low $\mathrm{C}_{5+}$ selectivity were reported before on $\mathrm{Co} /$ CNT catalysts, and this behavior was attributed to the small size of the cobalt nanoparticles. ${ }^{9}$ Nevertheless, colloidally synthesized monometallic cobalt catalysts were reported recently to have a very high long hydrocarbon chain selectivity 
a)

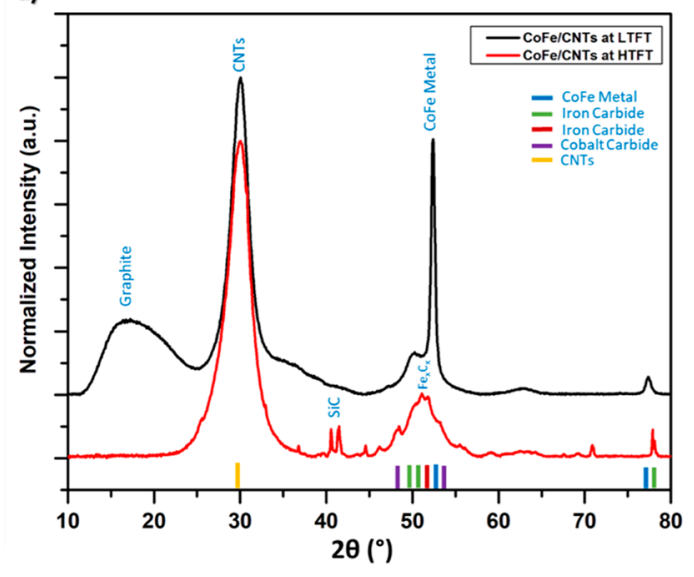

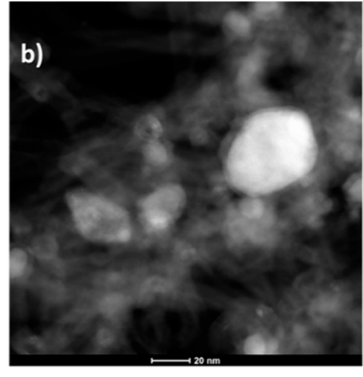
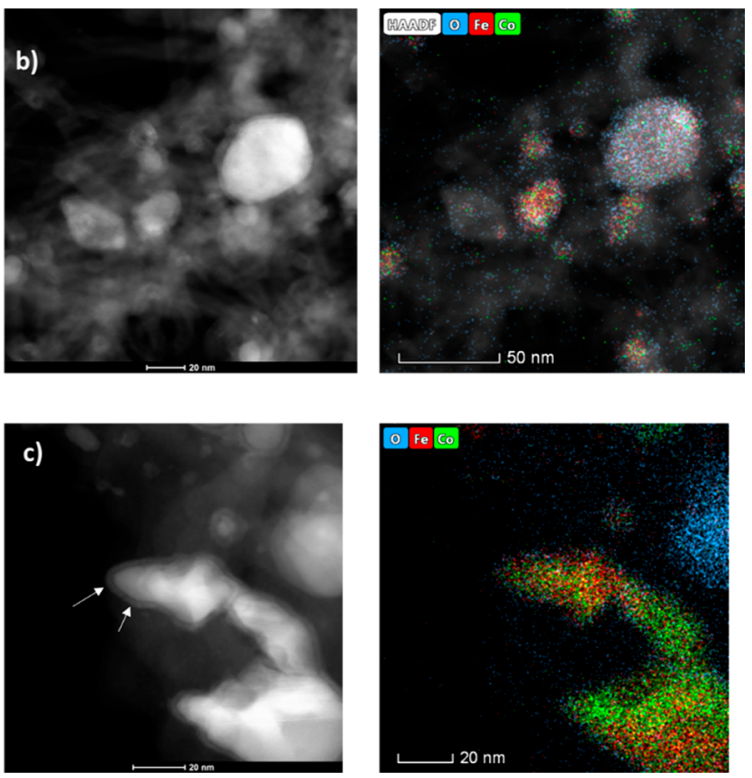

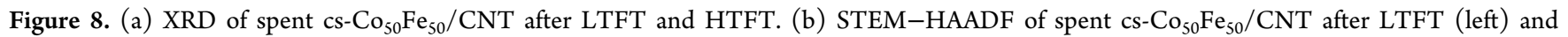
combined HAADF-EDX map of iron, cobalt, and oxygen in the same catalyst (right). (c) STEM-HAADF of spent cs-Co ${ }_{50} \mathrm{Fe}_{50} / \mathrm{CNT}$ after $\mathrm{HTFT}$ (left) and EDX map of iron, cobalt, and oxygen in the same catalyst (right).

at higher pressure operating conditions (20 bar) where the $\mathrm{CO}$ partial pressure is high enough to suppress methane formation and promote selectivity to $\mathrm{C}_{5+}$ products. $^{54}$

On the other hand, the colloidally synthesized monometallic iron and bimetallic cobalt iron catalysts showed the lowest methane selectivity at LTFT conditions (Figure $7 \mathrm{~b}$ ). It is possible that alloying iron and cobalt species in the bimetallic catalyst reduced the surface restructuring of cobalt species, which in turn lowered the possibility of formation of methane on the catalyst. As for the olefins' selectivity, iron showed the highest formation of olefins among the tested catalysts, which is expected and agrees with earlier reports on olefins' selectivity on iron at LTFT conditions. ${ }^{56}$ Finally, it is interesting to note that both bimetallic catalysts showed high selectivity for $\mathrm{C}_{5+}$ products, which we believe is attributed to the good nanoparticles distribution over the support that provides a suitable environment for growing long chain hydrocarbons, especially at LTFT conditions (Figure $7 \mathrm{~d}$ ). ${ }^{57}$ This could be better appreciated when observing that the $\mathrm{C}_{5+}$ hydrocarbons' selectivity in bimetallic catalysts at LTFT conditions is more than double that at HTFT conditions, which is understandable, knowing that the desorption probability of hydrocarbon intermediates from the catalyst surface increases with increasing reaction temperature. ${ }^{49}$

Furthermore, we observed that while the selectivity behavior of $\mathrm{cs}^{-\mathrm{Co}_{50}} \mathrm{Fe}_{50} / \mathrm{CNT}$ and $\mathrm{ci}-\mathrm{Co}_{50} \mathrm{Fe}_{50} / \mathrm{CNT}$ catalysts are similar toward the $\mathrm{C}_{2}-\mathrm{C}_{4}$ olefins formation, they show an opposite behavior toward methane and formation of $\mathrm{C}_{5+}$ hydrocarbons (Figure $7 \mathrm{~b}, \mathrm{~d}$ ). The $\mathrm{cs}^{-} \mathrm{Co}_{50} \mathrm{Fe}_{50} / \mathrm{CNT}$ shows clearly much higher formation of $\mathrm{C}_{5+}$ hydrocarbons $(\sim 42 \%)$ than ci- $\mathrm{Co}_{50} \mathrm{Fe}_{50} / \mathrm{CNT}(33 \%)$. In both catalysts, the selectivity to $\mathrm{C}_{5+}$ hydrocarbons decrease over time with the increase of methane formation during the course of catalysis, possibly because of the deactivation of the step edge sites in the catalysts.

The above observations during the LTFT and HTFT reactions show the strong synergism between iron and cobalt species in the bimetallic catalysts prepared by colloidal synthesis. It seems that during the FT, the Janus-like nanoalloy structure of cobalt and iron allows two types of chemistry taking places on both cobalt and iron active sites during FT. We can notice this from the high selectivity of the bimetallic catalyst to both $\mathrm{C}_{2}-\mathrm{C}_{4}$ olefins and $\mathrm{C}_{5+}$ hydrocarbons at HTFT and LTFT conditions, which highlights the role of different hydrocarbons chain growth sites on iron and cobalt in dictating the product selectivity in bimetallic system. On the basis of the observed catalytic behavior in the HTFT and LTFT conditions, we suggest that the surface of the reduced bimetallic catalysts in this study contained both iron and cobalt species. Therefore, a suitable future study of this bimetallic system would be to dope the iron crystal lattice with smaller concentrations of cobalt using colloidal synthesis and to study whether the catalytic behavior of this system will be similar or different with comparison to catalysts prepared by impregnation. We have successfully managed to utilize colloidal synthesis to make bimetallic $\mathrm{Co}_{10} \mathrm{Fe}_{90}$ nanocrystals and we found out that this concentration can be practically reached using this method (Figures S8 and S9).

On the basis of the differences in the catalytic activity and selectivity during LTFT and HTFT catalytic conditions, it is possible then to consider that changing the reaction temperature and conversion rate would dictate the formation of different active phases on the bimetallic species, which in turn would determine the hydrocarbons growth mechanism on the catalytic surface.

To test this hypothesis, we conducted XRD and TEM-EDX measurements on the spent catalysts. The XRD analysis showed two different diffraction patterns depending on the catalytic conditions (Figure 8a). The spent catalyst studied after LTFT showed mainly that the formation of cobalt-iron metallic species (at $2 \theta=53$ ) had a very similar pattern to those detected from the in situ XRD study discussed earlier. This indicated that the main active phase that had driven the lowtemperature FT catalysis was a metallic cobalt-iron alloys species. A small broad diffraction peak can also be observed at $2 \theta=50$, which can be either attributed to unreduced $\mathrm{Co}_{x} \mathrm{O}_{x} /$ 
$\mathrm{Fe}_{x} \mathrm{O}_{x}$ species (similar to these observed in the in situ XRD study) or cobalt and iron carbide species.

The XRD pattern of the spent catalyst after HTFT showed the absence of any metallic species' diffraction lines but rather multiple peaks representing mainly iron and cobalt carbide and oxide species. Diffraction lines of silica carbide particles residues used for catalyst dilution during HTFT can also be observed $(2 \theta=41)$.

TEM-EDX analysis of the spent catalysts generally agreed with the XRD observations. The TEM-EDX of the LTFT spent catalysts generally retained the cobalt and iron distribution within the nanoparticles, albeit with much less elemental distribution homogeneity as observed after the catalyst reduction (Figure $8 \mathrm{~b}$ and Figure S11). Another observation is that the oxygen distribution was mainly observed at the outer shell region of the nanoparticles, while very little carbon or oxygen was observed in the core region of the nanoparticles, which agrees with the XRD analysis of the catalyst and confirms that the FT active phase on the bimetallic catalyst is a cobalt-iron metallic alloy species. Finally, the particle size of the LTFT spent catalyst was found to range from 8 to $20 \mathrm{~nm}$.

On the other hand, TEM-EDX of the HTFT spent catalyst, showed the formation of an outer layer around the nanoparticles (Figure 8c). Analysis of the EDX elemental maps (Figure S12) confirmed that carbon was strongly deposited on the catalytic particles and less oxygen was observed on the particles, which indicates that carbon was strongly built up on the nanoparticles during the HTFT conditions and that the possible active phase in bimetallic catalysts is cobalt and iron carbides. Another difference is that the particles size of the HTFT catalyst was found to be much larger $(10-40 \mathrm{~nm})$ than that of the LTFT catalysts which demonstrates the effect of the high-temperature conditions on the nanoparticles aggregation.

The outcome of the XRD and TEM-EDX studies on the spent bimetallic catalysts clears the literature debate over the active phase of cobalt-iron species during Fischer-Tropsch and over whether a bimetallic alloy phase contributes to the activity and selectivity of certain products during FischerTropsch synthesis, since the study shows clearly that the nature of the active phase of the cobalt and iron species in their bimetallic catalysts seemed to depend mainly on the catalytic conditions that the catalysts operate in. Interestingly, we could not verify from the XRD or the TEM-EDX the formation of cobalt metallic species during HTFT, which is known to be the active phase of cobalt during Fischer-Tropsch reactions as mentioned earlier. Future mechanistic and characterization studies would be required to further determine how the interaction between to different metals in a bimetallic catalyst affects their active phase during FTS and how this would affect the FTS products.

Finally, reoxidation of the bimetallic catalyst would possibly regenerate the stable spinel structure of the $\mathrm{Co}-\mathrm{Fe}$ bimetallic catalyst, albeit with less monodispersed particle size due to the removal of the oleate organic ligands during the active metal attachment on the CNTs, which may affect the catalytic performance of the regenerated catalyst. ${ }^{58-60}$

\section{CONCLUSIONS}

We have reported the synthesis of carbon-nanotube-supported cobalt-iron bimetallic catalysts using a novel nonhydrolytic colloidal synthesis method. The cobalt-iron oxide nanocrystals were found to be homogeneously dispersed on the surface of the carbon nanotubes. We have also demonstrated that changing the cobalt-iron ratio and their metal loading in the supported catalyst is possible using this method. Highresolution electron microscopy studies of the bimetallic catalysts demonstrated the homogeneous distribution of iron and cobalt on the nanoparticles scale. Upon reduction, the cobalt iron oxide was found to be ultimately reduced to their metallic states via short-lived cobalt and iron monoxides. However, the complete reduction of all iron and cobalt species could not be confirmed. In addition, a CoFe nanoalloy was observed by studying individual reduced bimetallic nanoparticles, where cobalt and iron species were found to migrate to a cobalt/iron-rich regions inside the nanoparticles, forming a structure that is similar to Janus particles. EELS quantification of the reduced particles showed a thin interface $(2 \mathrm{~nm})$ between cobalt/iron-rich regions where an equal ratio of cobalt and iron coexists. As for the catalytic activity, we found that the bimetallic $\mathrm{Co}_{50} \mathrm{Fe}_{50} / \mathrm{CNT}$ had higher FischerTropsch activity than the monometallic iron catalyst and comparable activity to monometallic cobalt catalysts. Furthermore, its selectivity toward longer-chain hydrocarbons at HTFT and LTFT conditions was remarkable, which is attributed to the synergetic effects between cobalt and iron species in the bimetallic catalyst. Possible future studies on the effect of cobalt-iron alloying on the long-chain-hydrocarbon selectivity at low temperatures and higher syngas partial pressures (20 bar) might be interesting to test whether a similar selectivity trend would be observed on the colloidally synthesized bimetallic. Investigation of the spent catalysts showed that the active phase of the bimetallic species depends on the catalyst operation conditions. The spent catalysts retained their homogeneous distribution over the support after $42 \mathrm{~h}$ exposure to synthesis gas. This study demonstrates the importance of considering the effects of the chemical and structural dynamics of the bimetallic catalysts during the activation step on their overall catalytic performance.

\section{ASSOCIATED CONTENT}

\section{S Supporting Information}

The Supporting Information is available free of charge on the ACS Publications website at DOI: 10.1021/acscatal.8b04334.

Description of the synthesis methodology. TEM and high-resolution STEM-EELS analysis of CoFe/CNT catalysts with different cobalt to iron ratio. Detailed description of the characterization of CoFe/CNT catalysts prepared by incipient wetness impregnation. In situ XRD characterization of the reoxidation of the bimetallic catalysts (PDF)

\section{AUTHOR INFORMATION}

\section{Corresponding Author}

*E-mail: f.m.f.degroot@uu.nl.

ORCID $\odot$

Ahmed S. M. Ismail: 0000-0002-2282-1665

Marianna Casavola: 0000-0002-8902-7431

Tom W. van Deelen: 0000-0002-3666-4576

Frank M. F. de Groot: 0000-0002-1340-2186

\section{Notes}

The authors declare no competing financial interest. 


\section{ACKNOWLEDGMENTS}

The authors acknowledge the support provided by The Netherlands Center for Multiscale Catalytic Energy Conversion (MCEC), an NWO Gravitation programme funded by the Ministry of Education, Culture and Science of the government of The Netherlands.

\section{REFERENCES}

(1) Dry, M. E. High Quality Diesel via the Fischer-Tropsch Process - A Review. J. Chem. Technol. Biotechnol. 2002, 77, 43-50.

(2) Hagen, S.; Barfod, R.; Fehrmann, R.; Jacobsen, C. J. H.; Teunissen, H. T.; Chorkendoff, Ib Ammonia Synthesis with BariumPromoted Iron-Cobalt Alloys Supported on Carbon. J. Catal. 2003, 214, 327.

(3) Amelse, J. A.; Schwartz, L. H.; Butt, J. B. Iron Alloy FischerTropsch Catalysts: III. Conversion Dependence of Selectivity and Water-Gas Shift. J. Catal. 1981, 72, 95-110.

(4) Flahaut, E.; Govindaraj, A.; Peigney, A.; Laurent, C.; Rousset, A.; RAo, C. N. R. Synthesis of Single-Walled Carbon Nanotubes using Binary $(\mathrm{Fe}, \mathrm{Co}, \mathrm{Ni}$ ) Alloy Nanoparticles Prepared in Situ by the Reduction of Oxide Solid Solutions. Chem. Phys. Lett. 1999, 300, 236.

(5) Pham-Huu, C.; Keller, N.; Estournes, C.; Ehret, G.; Ledoux, M. J. Synthesis of $\mathrm{CoFe}_{2} \mathrm{O}_{4}$ Nanowire in Carbon Nanotubes. A New Use of the Confinement Effect. Chem. Commun. 2002, 17, 1882.

(6) Korneva, G.; Ye, H.; Gogotsi, Y.; Halverson, D.; Friedman, G.; Bradley, J.-C.; Kornev, K. G. Carbon Nanotubes Loaded with Magnetic Particles. Nano Lett. 2005, 5, 879-884.

(7) Schulz, H. Short History and Present Trends of FischerTropsch Synthesis. Appl. Catal., A 1999, 186, 3.

(8) Khodakov, A. Y. Fischer-Tropsch Synthesis: Relation between Structure of Cobalt Catalysts and Their Catalytic Performance. Catal. Today 2009, 144, 251-257.

(9) Bezemer, G. L.; Bitter, J. H.; Kuipers, H. P. C. E.; Oosterbeek, H.; Holewijn, J. E.; Xu, X.; Kapteijn, F.; Van Dillen, A. J.; De Jong, K. P. Cobalt Particle Size Effects in the Fischer-Tropsch Reaction Studied with Carbon Nanofiber Supported Catalysts. J. Am. Chem. Soc. 2006, 128, 3956-3964.

(10) Bligaard, T.; Nørskov, J. K.; Dahl, S.; Matthiesen, J.; Christensen, C. H.; Sehested, J. The Brønsted-Evans-Polanyi Relation and the Volcano Curve in Heterogeneous Catalysis. J. Catal. 2004, 224, 206.

(11) Calderone, V. R.; Shiju, N. R.; Ferre, D. C.; Rothenberg, G. Bimetallic Catalysts for the Fischer-Tropsch Reaction. Green Chem. 2011, 13, 1950-1959.

(12) Tavasoli, A.; Trépanier, M.; Malek Abbaslou, R. M.; Dalai, A. K.; Abatzoglou, N. Fischer- Tropsch Synthesis on Mono- and Bimetallic Co and Fe Catalysts Supported on Carbon Nanotubes. Fuel Process. Technol. 2009, 90, 1486-1494.

(13) Díaz, J. A.; Akhavan, H.; Romero, A.; Garcia-Minguillan, A. M.; Romero, R.; Giroir-Fendler, A.; Valverde, J. L. Cobalt and Iron Supported on Carbon Nanofibers as Catalysts for Fischer-Tropsch Synthesis. Fuel Process. Technol. 2014, 128, 417-424.

(14) Schwarz, J. A.; Contescu, C.; Contescu, A. Methods for Preparation of Catalytic Materials. Chem. Rev. 1995, 95 (3), 477-510.

(15) Handbook of Heterogeneous Catalysis; Ertel, G., Knözinger, H., Weitkamp, J, Eds.; VCH Verlagsgesellschaft mbH: Weiheim, German, 2008; Chapter 2.2.4-2.4.2, pp 467-484, DOI: 10.1002/ 9783527610044.hetcat0022.

(16) Yin, Y.; Alivisatos, A. P. Colloidal Nanocrystal Synthesis and the Organic-Inorganic Interface. Nature 2005, 437, 664-670.

(17) Pinna, N.; Neri, G.; Antonietti, M.; Niederberger, M. Nonaqueous Synthesis of Nanocrystalline Semiconducting Metal Oxides for Gas Sensing. Angew. Chem., Int. Ed. 2004, 43, 4345-4349.

(18) Talapin, D. V.; Lee, J. S.; Kovalenko, M. V.; Shevchenko, E. V. Prospects of Colloidal Nanocrystals for Electronic and Optoelectronic Applications. Chem. Rev. 2010, 110, 389-458.

(19) Shim, M.; Guyot-Sionnest, P. N-Type Colloidal Semiconductor Nanocrystals. Nature 2000, 407, 981-983.
(20) Fang, X.; Chen, C.; Jin, M.; Kuang, Q.; Xie, Z.; Xie, S.; Huang, R.; Zheng, L. Single-Crystal-Like Hematite Colloidal Nanocrystal Clusters: Synthesis and Applications in Gas Sensors, Photocatalysis and Water Treatment. J. Mater. Chem. 2009, 19, 6154-6160.

(21) Prieto, G.; Martinez, A.; Concepcion, P.; Moreno-Tost, R. Cobalt Particle Size Effects in Fischer-Tropsch Synthesis: Structural and in Situ Spectroscopic Characterization on Reverse MicelleSynthesized Co/ITQ-2 Model Catalysts. J. Catal. 2009, 266, 129.

(22) Chen, W.; Fan, Z.; Pan, X.; Bao, X. Effect of Confinement in Carbon Nanotubes on the Activity of Fischer-Tropsch Iron Catalyst. J. Am. Chem. Soc. 2008, 130 (29), 9414-9419.

(23) van Deelen, T. W.; Su, H.; Sommerdijk, N. A. J. M.; de Jong, K. P. Assembly and Activation of Supported Cobalt Nanocrystal Catalysts for the Fischer-Tropsch Synthesis. Chem. Commun. 2018, $54,2530-2533$.

(24) Tavasoli, A.; Abbaslou, R. M. M.; Trépanier, M.; Dalai, A. K. Fischer-Tropsch Synthesis Over Cobalt Catalyst Supported on Carbon Nanotubes in a Slurry Reactor. Appl. Catal., A 2008, 345, 134-142.

(25) Sun, S.; Zeng, H. Monodisperse $\mathrm{MFe}_{2} \mathrm{O}_{4}(\mathrm{M}=\mathrm{Fe}, \mathrm{Co}, \mathrm{Mn})$ Nanoparticles. J. Am. Chem. Soc. 2002, 124, 8204.

(26) Song, Q.; Zhang, Z. J. Shape Control and Associated Magnetic Properties of Spinel Cobalt Ferrite Nanocrystals. J. Am. Chem. Soc. 2004, 126 (19), 6164-6168.

(27) Casavola, M.; Hermannsdörfer, J.; de Jonge, N.; Dugulan, A. I.; de Jong, K. P. Fabrication of Fischer-Tropsch Catalysts by Deposition of Iron Nanocrystals on Carbon Nanotubes. Adv. Funct. Mater. 2015, 25, 5309-5319.

(28) Stéphan, O.; Gloter, A.; Imhoff, D.; Kociak, M.; Mory, C.; Suenaga, K.; Tencé, M.; Colliex, C. Electron Energy Loss Spectroscopy and Annular Dark Field Imaging at a Nanometer Resolution in a Scanning Transmission Electron Microscope. Surf. Rev. Lett. 2000, 07, 475 .

(29) Jeanguillaume, C.; Colliex, C. Spectrum-Image: The Next Step in EELS Digital Acquisition and Processing. Ultramicroscopy 1989, 28, 252.

(30) Ritz, B.; Heller, H.; Myalitsin, A.; Kornowski, A.; MartinMartinez, F. J.; Melchor, S.; Dobado, J. A.; Juarez, B. H.; Weller, H.; Klinke, C. Reversible Attachment of Platinum Alloy Nanoparticles to Nonfunctionalized Carbon Nanotubes. ACS Nano 2010, 4, 2438-44.

(31) Li, Z.; Lu, C.; Xia, Z.; Zhou, Y.; Luo, Z. X-ray.X-Ray Diffraction Patterns of Graphite and Turbostratic Carbon. Carbon 2007, 45, $1686-1695$

(32) Mohamed, R. M.; Rashad, M. M.; Haraz, F. A.; Sigmund, W. Structure and Magnetic Properties of Nanocrystalline Cobalt Ferrite Powders Synthesized using Organic Acid Precursor Method. J. Magn. Magn. Mater. 2010, 322, 2058-2064.

(33) Logdberg, S.; Tristantini, D.; Borg, O.; Ilver, L.; Gevert, B.; Jaras, S.; Blekkan, E. A.; Holmen, A. Hydrocarbon Production via Fischer-Tropsch Synthesis from $\mathrm{H}_{2}$-Poor Syngas Over Different $\mathrm{Fe}$ $\mathrm{Co} / \gamma-\mathrm{Al}_{2} \mathrm{O}_{3}$ Bimetallic. Appl. Catal., B 2009, 89, 167-182.

(34) Griboval-Constant, A.; Butel, A.; Ordomsky, V. V.; Chernavskii, P. A.; Khodakov, A. Y. Cobalt and Iron Species in Alumina Supported Bimetallic Catalysts for Fischer-Tropsch Reaction. Appl. Catal., A 2014, 481, 116-126.

(35) Hunt, J. A.; Williams, D. B. Electron Energy-Loss SpectrumImaging. Ultramicroscopy 1991, 38, 47-73.

(36) Lucas, G.; Burdet, P.; Cantoni, M.; Hébert, C. Multivariate Statistical Analysis as a Tool for The Segmentation of 3D Spectral Data. Micron 2013, 52-53, 49-56.

(37) De la Pena O'Shea, V. A.; Alvarez-Galvan, M. C.; CamposMartin, J. M.; Menendez, N. N.; Tornero, J. D.; Fierro, J. L. G. Surface and Structural Features of Co-Fe Oxide Nanoparticles Deposited on a Silica Substrate. Eur. J. Inorg. Chem. 2006, 2006, 5057-5068.

(38) Ma, X.; Sun, Q.; Ying, W.; Fang, D. Effects of Promoters on Catalytic Performance of Fe-Co/SiO2 Catalyst for Fischer-Tropsch Synthesis. J. Nat. Gas Chem. 2009, 18, 354-358.

(39) Ma, X.; Sun, Q.; Cao, F.; Ying, W.; Fang, D. Effects of the Different Supports on the Activity and Selectivity of Iron-Cobalt 
Bimetallic Catalyst for Fischer-Tropsch Synthesis. J. Nat. Gas Chem. 2006, 15, 335-339.

(40) de la Pena O’Shea, V. A.; Menéndez, N. N.; Tornero, J. D.; Fierro, J. L. G. Unusually High Selectivity to $\mathrm{C}_{2+}$ Alcohols on Bimetallic CoFe Catalysts During CO Hydrogenation. Catal. Lett. 2003, 88, 123-128.

(41) De la Peña O’Shea, V. A.; Álvarez-Galván, M. C.; CamposMartín, J. M.; Fierro, J. L. G. Fischer-Tropsch Synthesis on Monoand Bimetallic $\mathrm{Co}$ and $\mathrm{Fe}$ Catalysts in Fixed Bed and Slurry Reactors. Appl. Catal., A 2007, 326, 65-73.

(42) Tihay, F.; Pourroy, G.; Richard-Plouet, M.; Roger, A. C.; Kiennemann, A. Effect of Fischer-Tropsch Synthesis on the Microstructure of $\mathrm{Fe}-\mathrm{Co}$-Based Metal/Spinel Composite Materials. Appl. Catal., A 2001, 206, 29-42.

(43) Rez, P.; Res, J. Accurate Cross Sections for Microanalysis. J. Res. Natl. Inst. Stand. Technol. 2002, 107, 487.

(44) Manoubi, T.; Tencé, T.; Walls, M. G.; Colliex, C. Curve Fitting Methods for Quantitative Analysis in Electron Energy Loss Spectroscopy. Microsc., Microanal., Microstruct. 1990, 1, 23-39.

(45) Calvo, F. Thermodynamics of nanoalloys. Phys. Chem. Chem. Phys. 2015, 17, 27922.

(46) Zhang, J.; Muller, J. O.; Zheng, W.; Wang, D.; Su, D.; Schlogl, R. Individual Fe-Co Alloy Nanoparticles on Carbon Nanotubes: Structural and Catalytic Properties. Nano Lett. 2008, 8, 2738.

(47) Torres Galvis, H. M.; Bitter, J. H.; Davidian, T.; Ruitenbeek, M.; Dugulan, A. I.; de Jong, K. P. Iron Particle Size Effects for Direct Production of Lower Olefins from Synthesis Gas. J. Am. Chem. Soc. 2012, 134, 16207-16215.

(48) Torres Galvis, H. M.; Bitter, J. H.; Khare, C. B.; Ruitenbeek, M.; Dugulan, A. I.; de Jong, K. P. Supported Iron Nanoparticles as Catalysts for Sustainable Production of Lower Olefins. Science 2012, $335,835-838$.

(49) Schulz, H. Comparing Fischer-Tropsch Synthesis on Iron- and Cobalt Catalysts-the Dynamics of Structure and Function in Fischer-Tropsch Synthesis, Catalysts and Catalysis. Stud. Surf. Sci. Catal. 2007, 163, 177-199.

(50) Torres Galvis, H. M.; Koeken, A. C. J.; Bitter, J. H.; Davidian, T.; Ruitenbeek, M.; Dugulan, A. I.; de Jong, K. P. Effect of Precursor on the Catalytic Performance of Supported Iron Catalysts for the Fischer-Tropsch Synthesis of Lower Olefins. Catal. Today 2013, 215, 95-102.

(51) Arai, H.; Mitsuishi, K.; Seiyama, T. TiO 2 -Supported Fe-Co, Co$\mathrm{Ni}$, and Ni-Fe Alloy Catalysts for Fischer-Tropsch Synthesis. Chem. Lett. 1984, 13, 1291-1294.

(52) Eschemann, T. O.; Lamme, W. S.; Manchester, R. L.; Parmentier, T. E.; Cognigni, A.; Rønning, M.; de Jong, K. P. Effect of Support Surface Treatment on the Synthesis, Structure, and Performance of Co/CNT Fischer-Tropsch catalysts. J. Catal. 2015, $328,130-138$.

(53) Zhang, H.; Lancelot, C.; Chu, W.; Hong, J.; Khodakov, A. Y.; Chernavskii, P. A.; Zheng, J.; Tong, D. The Nature of Cobalt Species in Carbon Nanotubes and Their Catalytic Performance in FischerTropsch Reaction. J. Mater. Chem. 2009, 19, 9241-9249.

(54) van Deelen, T. W.; Nijhuis, J. J.; Krans, N. A.; Zečević, J.; de Jong, K. P. Preparation of Cobalt Nanocrystals Supported on Metal Oxides to Study Particle Growth in Fischer-Tropsch Catalysts. ACS Catal. 2018, 8, 10581-10589.

(55) Iglesia, E. Design, Synthesis, and Use of Cobalt-Based FischerTropsch Synthesis Catalysts. Appl. Catal., A 1997, 161, 59.

(56) Xu, K.; Sun, B.; Lin, J.; Wen, W.; Pei, Y.; Yan, S. R.; Qiao, M. H.; Zhang, X. X.; Zong, B. N. $\varepsilon$-Iron Carbide as a Low-Temperature Fischer-Tropsch Synthesis Catalyst. Nat. Commun. 2014, 5, 5783.

(57) Jager, B.; Espinoza, R. Advances in Low Temperature FischerTropsch Synthesis. Catal. Today 1995, 23 (1), 17.

(58) Saib, A. M.; Moodley, D. J.; Ciobîcă, I. M.; Hauman, M. M.; Sigwebela, B. H.; Weststrate, C. J.; Niemantsverdriet, J. W.; van de Loosdrecht, J. Fundamental Understanding of Deactivation and Regeneration of Cobalt Fischer-Tropsch Synthesis Catalysts. Catal. Today 2010, 154, 271-282.
(59) Nakhaei Pour, A.; Housaindokht, M. R. Fischer-Tropsch Synthesis on Iron Catalyst Promoted With HZSM-5 Zeolite: Regeneration Studies of Catalyst. J. Nat. Gas Sci. Eng. 2013, 14, $49-54$.

(60) Sonal; Kondamudi, K.; Pant, K. K.; Upadhyayula, S. Synergistic Effect of Fe-Co Bimetallic Catalyst on FTS and WGS Activity in the Fischer-Tropsch Process: a Kinetic Study. Ind. Eng. Chem. Res. 2017, $56,4659-4671$. 